\title{
Novel mtDNA Imparts the Connective Tissue Disorder of a Tourette Pedigree
}

Patrick M. Schaefer, Dr. rer. nat. ${ }^{1}$, Leonardo Scherer Alves, Ph.D. ${ }^{1}$, Maria Lvova, M.D. ${ }^{1}$, Jessica Huang ${ }^{1}$, Komal Rathi, MSc ${ }^{2}$, Kevin Janssen ${ }^{1}$, Ph.D., Arrienne Butic ${ }^{1}$, Tal Yardeni, Ph.D. ${ }^{1}$, Ryan Morrow, Ph.D. ${ }^{1}$, Marie Lott, M.A. ${ }^{1}$, Kierstin Keller, C.G.C. ${ }^{1}$, Benjamin A. Garcia, Ph.D. ${ }^{3}$, Clair A. Francomano, M.D. ${ }^{4}$, Douglas C. Wallace, Ph.D. ${ }^{1,5^{*}}$.

${ }^{1}$ Center for Mitochondrial and Epigenomic Medicine, Children's Hospital of Philadelphia, PA, 19104.

2 Department of Biomedical Informatics, Children's Hospital of Philadelphia, PA, 19104

${ }^{3}$ Department of Biochemistry and Molecular Biophysics, Washington University School of Medicine, St. Louis, MO, USA

${ }^{4}$ Department of Medical and Molecular Genetics, Indiana University School of Medicine, Indianapolis, IN, USA

${ }^{5}$ Department of Pediatrics, Division of Human Genetics, Perelman School of Medicine, University of Pennsylvania, Philadelphia, PA, 19104.

* Corresponding author: wallaced1@chop.edu

\section{Abstract}

Mitochondrial dysfunction is associated with a range of clinical manifestations including neuropsychiatric and metabolic disorder. Here, we reanalyzed a family with an L-Histidine Decarboxylase (HDC) variant previously linked to Tourette syndrome but with associated connective tissue and metabolic features of unknown etiology. We identified a mitochondrial haplogroup J-defining mutation on the haplogroup $\mathrm{H}$ background that functionally interacts with the L-Histidine Decarboxylase variant via calcium homeostasis. Our findings establish how a common mtDNA variant on a different mtDNA background can result in mitochondrial dysfunction, demonstrate a role for histaminergic signaling in modifying mitochondrial phenotypes, and link mitochondria dysfunction to connective tissue phenotypes. 
Mitochondria are increasingly recognized as integral factors in many pathologies, including diabetes or neuropsychiatric disorders (1). While most components of the mitochondria are nuclear-encoded, the maternally inherited mitochondrial DNA (mtDNA) still retains 13 essential subunits of oxidative phosphorylation (OxPhos) as well as the structural RNAs for the mitochondrial protein synthesis machinery (2-4). During human evolution, functional mtDNA variants arose along radiating maternal lineages that resulted in subtle changes in mitochondrial bioenergetics. These permitted humans to adapt to the new environments they encountered as they migrated out-of-Africa and around the globe. These adaptive variants function within the context of pre-existing mtDNA functional variants resulting in an integrated bioenergetic state. Those variant combinations that proved regionally beneficial became enriched to form geographically constrained groups of related haplotypes, known as a haplogroup (5).

Tourette syndrome is an autism spectrum disorder (ASD) characterized by motor and vocal tics with a strong genetic component, and a mtDNA mutant has been shown to produce ASD-like symptoms in a mouse model (6). In a large two-generation pedigree, linkage analysis identified a null variant in the LHistidine Decarboxylase (HDC) gene (W317X) as a candidate for causing Tourette syndrome (7). However, this pedigree manifests several unusual characteristics that cannot be explained by Mendelian inheritance of the HDC gene variant. First, a paternal heterozygous variant was passed to all 8 living offspring, while seven other conceptions were lost as miscarriages. The probability of this chain of events to happen by chance is $0.4 \%$. Second, the mother and all living offspring display a complex metabolic phenotype in association with a skeletal and connective tissue disorder that resembles Ehlers-Danlos syndrome.

We have tested the hypothesis that this pedigree involves a deleterious maternally-inherited mtDNA for which the HDC variant was protective. This resulted in only the offspring that inherited the HDC variant surviving, while still manifesting the metabolic and connective tissue defects associated with the deleterious mtDNA. 


\section{Results}

\section{Family harbors a dysfunctional mtDNA variant}

We reevaluated, assembled, and summarized the clinical data for the two-generation pedigree of Tourette syndrome and obtained additional data from subsequent family assessments (7). In addition to a range of neurological symptoms (Tourette, Obsessive-Compulsive Disorder (OCD), ASD) reported in the original paper (7), additional information was identified for the mother (Fig.1a, 12) and her offspring. This elaborated on the previously reported information pertaining to skeletal and connective tissue manifestations, including Chiari malformation, cranial-cervical instability, hypermobility of joints, skin herniation, wound dehiscence, aortic valve disease, ankylosing spondylitis, diabetes, fatigability, and dysautonomic manifestations (see Appendix). The apparent matrilineal transmission of this complex array of clinical manifestations in all 8 offspring suggested a mtDNA disorder. Our resulting interpretation of the two-generation pedigree is summarized in Fig.1a.

To determine if there was a mtDNA contribution to this pedigree, we sequenced the mtDNA of the mother and five of her children, their mtDNA variants being presented in Fig.S1a. The mtDNA haplogroup is H7, but this family's mtDNA also contains the haplogroup J-defining variant ND5 m.13708G>A (codon 458 Alanine to Threonine). This is noteworthy since the ND5 m.13708G >A is very rarely associated with haplogroup H7 ( $\mathrm{AF}=0.86 \%, 3 / 348)$ in Mitomap (8) and rarely on other haplogroup H mtDNAs (Fig.S1b, c).

To assess whether the ND5 m.13708G>A variant on haplogroup $\mathrm{H} 7$ affects mitochondrial function, we created transmitochondrial cybrids that have the same $143 \mathrm{~B}\left(\mathrm{TK}^{-}\right)$cell nDNA but harbor either the patient mtDNA (mutant) or the mtDNA of a haplogroup-matched normal control (control) (Fig.S1a, Fig.S2a). We observed a lower electron transport system capacity with reduced complex I and II respiration in the mutant cybrids (Fig.1b, Fig.S2b, d). In line with the lower respiration, the mutant cybrids displayed a reduced mitochondrial membrane potential (Fig.1c) and NAD $/ \mathrm{NADH}$ redox ratio (Fig.1d). In addition, 
both mitochondrial and cytosolic ROS levels were increased (Fig.1e, Fig.S2e), doubling time was longer

(Fig.1f) and apoptosis was increased (Fig.S2f) in the mutant cybrids. In contrast, haplogroup J cybrids harboring the ND5 m.13708G $>$ A variant in its standard context showed similar respiration haplogroup $\mathrm{H}$ cybrids and even reduced ROS production (Fig.S3). Hence, when arising in the wrong context, the ND5 m.13708G $>$ A variant is incompatible with the resident haplogroup $\mathrm{H}$ mtDNA functional variants resulting in mitochondrial dysfunction and clinical manifestations.

To further evaluate the impact of the ND5 m.13708G>A-H7 mtDNA mutant on cellular physiology, we performed multi-omics on the cybrids. This revealed profound changes to the gene expression, histone acetylation, and metabolite profile (Fig.2).

RNA sequencing revealed downregulation of OxPhos and nicotinamide metabolism in mutant cybrids (Fig.2a, Fig.S4). This goes along with a more reduced nuclear NAD $/$ NADH redox ratio (Fig.2b) and altered nucleotide levels and TCA cycle intermediates in the mutant cybrids (Fig.S4b, c). Interestingly, while nicotinamide levels were increased, 6-methylnicotinamide was significantly decreased in the mutant cybrids (Fig.S4b), indicating a problem in the SAM-dependent degradation of nicotinamide. Global metabolomics point to alterations in methionine metabolism and homocysteine degradation (Fig.2c) and RNA sequencing revealed a downregulation of the one carbon metabolism (Fig.2a, Fig.S4d). Targeted metabolomics verified reduced homocysteine levels but increased cystathionine levels and trends towards a reduced SAM/SAH and GSSG/GSH ratio in the mutant cybrids (Fig.S4e), revealing a defect in the mitochondrial one carbon metabolism. Consistent with lower methyl-donor availability, we detected histone hypomethylation in the mutant cybrids (Fig.2d, Fig.S5). In addition, global metabolomics revealed increased levels of acylcarnitines (Fig.2c, S6d) in the mutant cybrids, indicating alterations in fatty acid metabolism. This is further supported by significant changes to the ketone body metabolism and an upregulation of the cholesterol biosynthesis pathway in RNA sequencing (Fig.S6a,b). Importantly, RNAseq revealed significant differential expression of genes associated with collagen formation and 
neurodevelopment in the patient mtDNA cybrids (Fig.2a, Fig.S6c), linking the mitochondrial variant with the connective tissue phenotype and Tourette syndrome of the family.

Taken together, the ND5 m.13708G>A variant on the haplogroup $\mathrm{H} 7$ background affects mitochondrial fatty acid metabolism, one carbon metabolism, nicotinamide metabolism and collagen metabolism, thus profoundly changing cellular gene expression and physiology.

\section{HDC variant acts as a beneficial nuclear modifier via calcium homeostasis}

Next, we asked whether the heterozygous HDC variant from the father could have a beneficial effect on the maternal ND5 m.13708G>A-H7 mtDNA, thus explaining why all living offspring carry the HDC variant while seven others died in utero. Given the central role of HDC in histamine signaling (9) and subsequent calcium release from the ER (10), we assessed calcium homeostasis in the cybrids.

We found higher cytosolic calcium but reduced mitochondrial calcium levels in the mtDNA mutant cybrids at baseline (Fig.3a). Histamine-induced calcium release from the ER resulted in a stronger increase of cytosolic calcium in the mutant cybrids (Fig.3b), which was weakened but retained upon pretreatment with a mitochondrial calcium uptake inhibitor (Fig.S7c) but not after thapsigargin-induced calcium release

(Fig.S7d). This demonstrates an increased histamine sensitivity in the mutant cybrids, which corresponds to an upregulation of the histamine 1 receptor signaling pathway on the RNA level (Fig.S7e). At the same time, the partial rescue with the MCU inhibitor indicates a lower mitochondrial calcium uptake in the mutant cybrids. This is confirmed by a lower increase of mitochondrial calcium upon histamine treatment (Fig.S7b) and downregulation of the mitochondrial calcium ion transport (Fig.S7a) in the mutant cybrids. Taken together, the ND5 m.13708G>A variant on haplogroup H7 background alters calcium homeostasis and histamine signaling, providing a link to the HDC variant.

To simulate an increased HDC activity, we exposed the cybrids to histamine for $24 \mathrm{~h}$. The m.13798G $>\mathrm{A}-$ H7 mutant but not control cybrids showed a further reduced respiration (Fig.3c, Fig.S8a) and increased 
ROS production (Fig.3d) after treatment with $100 \mu \mathrm{M}$ histamine for $24 \mathrm{~h}$. This could be blocked by simultaneous treatment with the histamine 1 receptor antagonist pyrilamine (Fig.S8c-e). In addition, we found an increased mitochondrial permeability transition pore (mPTP) opening in the mutant cybrids (Fig.S8b), indicating that a mitochondrial calcium overload induced via histamine 1 receptor-mediated calcium release from the ER contributes to mutant cybrid cell death (Fig.3e). Pyrilamine inhibition of the histamine 1 receptor slightly reduced mitochondrial respiration in controls but mitigated the mitochondrial phenotypic difference between mutant and control cybrids (Fig.S9b-h). In summary, lower histamine levels due to the paternal HDC variant mitigate the increased calcium overload toxicity for the ND5 m.13708G>A variant on the H7 background, thus explaining why all of the surviving offspring inherited the HDC mutant allele.

The remaining question is why the mother, who does not carry the HDC variant, was affected with the metabolic and connective tissue disorders but was still alive. One possibility is that the mother harbored a nuclear genotype that was protective of the ND5 m.13708G $>$ A-H7 variant mitochondrial defect. To assess if the mother's genotype affected her mitochondrial physiology, we created immortalized lymphoblasts from the mother, 4 of her children and 3 haplogroup-matched controls. For the mother and her children, we found no changes in respiratory capacity or calcium handling compared to controls, though a switch from complex I to complex II respiration and increased cytosolic ROS was observed (Fig.4a-d). This mild mitochondrial phenotype compared to the mutant cybrids might be explained by low and thus undetectable expression of HDC and histamine 1 receptor in the lymphoblasts, similar to histamine 1 receptor inhibition in cybrids (Fig.S9).

Comparing the mother's mitochondrial physiology to that of her children (Fig.4e-h), we found that the lymphoblasts of all children but not the mother had increased ROS levels compared to control lymphoblasts (Fig.4g, 12). Since increased ROS predisposes to apoptosis, the mother may harbor a multiple allele antioxidant genotype not transmitted to her offspring. 
To verify if lowering ROS could mitigate the mother's phenotype, we treated the ND5 m.13708G>A-H7 cybrids with $\mathrm{N}$-Acetyl-cysteine (NAC) and nicotinamide riboside (NR). Both partially rescued the mitochondrial defect in the mutant cybrids, resulting in a shorter doubling time, higher respiration, and lower cytosolic ROS levels (Fig.S10). This not only supports the mother harboring an enhanced antioxidant genotype but also suggests new therapeutic approaches for this family and perhaps other patients with mitochondrially-based connective tissue disorders.

\section{Discussion}

We reanalyzed an unusual family with familial Tourette syndrome, metabolic dysfunction, and connective tissue disorder. Mendelian inheritance of a heterozygous HDC variant alone could not explain why all eight living children carry the HDC variant, suggesting that the seven additional miscarriages were the conceptuses that inherited the father's normal HDC allele. In identifying an out-of-context mtDNA mutant in the patient family, we gained three crucial insights into the diversity of mitochondrial disorders and the mitochondrial etiology of the Tourette pedigrees patient phenotypes.

First, we could establish that a common haplogroup J-defining mtDNA variant causes mitochondrial dysfunction when combined with haplogroup $\mathrm{H} 7$, while the same variant in haplogroup J does not affect bioenergetics. This points to an incompatibility of ND5 m.13708G>A variant with mtDNA variants present in haplogroup H7. Incompatibility between haplogroup-associated mtDNA variants may explain why the mtDNA is strictly maternally inherited, thus blocking inter-haplogroup recombination and the mixing of incompatible variant alleles (2). The existence of functional alterations in mtDNAs has been documented when mtDNAs of different origins have been mixed within a mouse and when different mtDNAs are placed on the same nDNA background (11-14). While the exact molecular basis of the intra-mtDNA ND5 m.13708G >A haplogroup H7 incompatibility is unknown, one might hypothesize that mitochondrial electron transfer is hampered, resulting in lower respiration and higher ROS production. Taken together, 
we demonstrate that cross mtDNA mixing between common mtDNA variants can result in incompatibility and mitochondrial disorders. Clinically, this implies the need for not only checking the frequency of mtDNA variants but also the frequency of co-occurrence with other mtDNA variants when evaluating mtDNAs for pathological relevance.

Second, we demonstrate a mitochondrial-nuclear interaction with the paternal HDC variant and maternal mtDNA by establishing an important role for histaminergic signaling in modifying the mitochondrial phenotype. We showed that the HDC null allele (W317X) acts as a beneficial nuclear modifier for the deleterious maternal mtDNA. Given the crucial role for calcium signaling in embryogenesis (15), it seems plausible that the seven miscarriages were a result of a non-compensated dysregulation in calcium homeostasis due to the presence of the maternal mtDNA but absence of the HDC variant. While the mother herself does not harbor the HDC variant, we found lower cytosolic ROS levels in her lymphoblastoid cells compared to those of her children, suggesting the presence of additional nuclear antioxidant modifiers. Consistent with previous reports for mitochondrial dysfunction in cells (16) or mice (6) lowering the ROS levels in the ND5 m.13708G>A-H7 cybrids rescued the mitochondrial phenotype.

Third, we revealed a putative mitochondrial etiology for the connective tissue phenotype of the patients and potential involvement in the Tourette syndrome. The connective tissue phenotype of the family resembles Ehlers Danlos Syndrome, which is commonly caused by mutations in collagen, other elements of the connective tissue, or the enzymes involved in processing the structural proteins of the extracellular matrix (17). It has been shown that collagen VI deficiency results in mitochondrial dysfunction, including calcium deregulation and mPTP opening in mice with a form of muscular dystrophy (18). Interestingly, mPTP desensitization improved the muscular dystrophy, suggesting a bidirectional relationship (19). Here we provide direct evidence that mitochondrial dysfunction alters collagen and extracellular matrix gene expression resulting in the connective tissue disorder. Mechanistically, it has been suggested that 
mitochondria could regulate collagen metabolism via the redox state (20), which is significantly altered in the patient cybrids.

With respect to the underlying cause of the neurological phenotypes in this family, the HDC variant may well be the cause of the Tourette phenotype (7), but the mtDNA variant causes the connective tissue and metabolic phenotypes. This perspective is supported by the fact that the father but not the mother displays a Tourette phenotype and by additional studies in recent years that linked the HDC variant to Tourette syndrome $(21,22)$. However, the extended neurological manifestations and the skewed inheritance of the HDC gene in the surviving offspring cannot be explained solely by inheritance of the HDC gene. Rather, the mtDNA defects must also contribute to the diverse neurological symptoms of the offspring as well as the connective tissue and metabolic manifestations of the mother and her children. It has already been shown that a mtDNA mutation is sufficient to cause ASD-like symptoms in mice (6) and mitochondrial dysfunction is thought to be a key feature in neurodegenerative disorders (23). RNASeq analysis of the patient transmitochondrial cybrids identified the dysregulation of genes associated with neurodevelopment and axon guidance. In addition, we showed that histamine aggravates the mitochondrial phenotype in vitro. Given the prominent role of histaminergic signaling in the brain, it is possible that both the HDC variant as well as the mtDNA variant account for the inheritance of the neuropsychological disorders of the children. The beneficial effects of $\mathrm{N}$-acetylcysteine and nicotinamide riboside on the mitochondrial dysfunction of these patients suggests potential therapeutic options, some of which might be generalizable to other mitochondrially-related neuropsychiatric and/or connective tissue disorders.

\section{Online Methods}

\section{mtDNA Sequencing}


The entire mtDNA was PCR amplified in two fragments using the SequalPrep Long PCR Kit (Invitrogen \#A10498) with the following primers: (1) hmtL9611: 5'-TCCCACTCCTAAACACATCC-3', hmtH1405: 5'ATCCACCTTCGACCCTTAAG-3'; and (2) hmtL1305: 5'-GTAAGCGCAAGTACCCACG-3', hmtH9819: 5'GCCAATAATGACGTGAAGTCC-3'. The PCR products were visualized on an agarose gel with ethidium bromide to assess amplification and product size specificity. For library preparation the two PCR fragments were combined in equal ratios to make 200 ng of DNA, fragmented and blunt-end ligated to 3' P1 and barcoded 5' A-BC adaptors (Life Technologies) using the NEBNext Fast DNA Fragmentation \& Library Prep Set for Ion Torrent (New England Biolabs \#E6285S/L). Size selection was done using Agencourt AMPure XP beads (Beckman Coulter). Ready amplified libraries were quantified with the Ion Library Quantitation kit (Life Technologies \#4468802). Multiple barcoded samples per run were pooled in equimolar ratios and templated Ion Sphere Particles (ISPs) were prepared and enriched on the One Touch 2 and ES systems using the lon PGM Template OT2 200 kit (Life Technologies \#4480974). The enriched templated ISPs were then loaded on an lon 318 sequencing chip and sequenced on an lon Personal Genome Machine (PGM) using Ion PGM Sequencing 200 v2 kit (Life Technologies). Data was analyzed using the Ion Torrent Suite v4.0.1 and NextGENe software v2.3.3. Haplogroups were identified using the plugin MtDNA Variant Caller 4.4.3.3 and the HaploGrep algorithm.

\section{Cell lines and treatments}

Venous blood samples were obtained from the patient family and three controls following informed consent. Leukocytes were isolated from whole blood by Ficoll-Hypaque gradients and the isolated cells were EBV transformed to establish lymphoblast cell lines by Corriell. Lymphoblasts were maintained in RPMI 1640 medium (11879, Gibco) supplemented with 10\% heat-inactivated fetal calf serum, $1 \mathrm{mM}$ glucose, and $2 \mathrm{mM}$ uridine. For all lymphoblastoid cell line experimental procedures, 4 million cells were seeded in $15 \mathrm{ml}$ of culture media $48 \mathrm{~h}$ prior to the experiment. 
Transmitochondrial cybrids were created from the most affected daughter (Fig. 1, II5) and the closestmatching control. Lymphoblasts were enucleated with actinomycin $D(0.5 \mu \mathrm{g} / \mathrm{mL})$ for $14 \mathrm{hrs}$ and then fused with $143 \mathrm{~B}(\mathrm{TK}-) \rho^{0}$ osteosarcoma cells using $45 \%$ polyethylene glycol/DMSO. Cells were plated and grown under selection for 4 weeks in DMEM containing $10 \%$ dialyzed FBS, $50 \mu \mathrm{g} / \mathrm{mL} \mathrm{BrdU}$, and no uridine (24). Clones were picked and grown separately as individual cell lines, resulting in 2 clones of patient cybrids harboring the patient mtDNA and 1 clone of control cybrids harboring the mtDNA of the closestmatching control. Both patient clones were used for the biochemical and bioenergetic comparison to control cybrids, and showed very similar responses. The results were pooled for statistical and graphical representation.

All cybrids were maintained in DMEM medium (11966, Gibco) supplemented with $10 \%$ heat-inactivated fetal calf serum, $1 \mathrm{mM}$ glucose and $2 \mathrm{mM}$ uridine. For all experimental procedures, 2.5 million cells were seeded in T75 cell culture flasks in $10 \mathrm{ml}$ culture media $48 \mathrm{~h}$ prior to the experiment.

For histamine treatment, a $100 \mathrm{mM}$ histamine stock solution in water was prepared fresh the day of use $100 \mu \mathrm{M}$ histamine was added to the cells either $24 \mathrm{~h}$ prior or immediately prior to the respective measurements.

For pyrilamine treatment, a $100 \mathrm{mM}$ pyrilamine stock solution in PBS was prepared and stored at $-20^{\circ} \mathrm{C}$. $100 \mu \mathrm{M}$ pyrilamine was added to the cells $24 \mathrm{~h}$ prior to the respective measurements and before histamine addition.

For thapsigargin treatment, a $1 \mathrm{mM}$ thapsigargin stock solution in DMSO was prepared and stored at $20^{\circ} \mathrm{C} .2 \mu \mathrm{M}$ thapsigargin was added to the cells immediately prior to the measurements.

For the MCU inhibitor treatment with KB-R7943, a 5 mM KB-R7943 stock solution in DMSO was prepared and stored at $-20^{\circ} \mathrm{C} .10 \mu \mathrm{M} \mathrm{KB}-\mathrm{R} 7943$ was added to the cells 5 min prior to the measurements. 
For nicotinamide riboside (NR) and $\mathrm{N}$-acetyl-cysteine (NAC) treatments, the culture media was supplemented with $300 \mu \mathrm{M}$ NR or $1 \mathrm{mM}$ NAC and cells were cultured in the continuous presence of both compounds. Representative measurements were started 1 week after initiation of the treatments and continued for 3 weeks. All parameters assessed were checked for trends coinciding with treatment duration, but cellular physiology remained stable.

\section{Doubling time}

The doubling time was determined in conjunction with the respirometry and flow experiments. Cells were harvested and counted for respirometry/flow cytometry. This allowed calculation of the doubling time via the formula:

Doubling time $(\mathrm{h})=$ culture time $(\mathrm{h}) / \log _{2}\left(\right.$ cell $_{\text {number }}$ end $/$ cell number $\left._{\text {start }}\right)$

\section{High Resolution respirometry in intact cells}

Respiration in intact cells was measured as described (25). Briefly, the Oroboros Oxygraph-2k was first calibrated to air with the respective cell culture medium of the cells to be measured (block temperature: $37^{\circ} \mathrm{C}$, stirrer speed $=750 \mathrm{rpm}$, oxygen sensor gain $=4$, data recording interval $2 \mathrm{sec}$ ). Subsequently, the cells were harvested and 2 million cells, resuspended in their conditioned medium, were loaded into each chamber of the respirometer. The first oxygen consumption plateau corresponds to Routine respiration, determining respiration under endogenous substrates and energy demand. Addition of $0.5 \mu \mathrm{l}$ of $5 \mathrm{mM}$ oligomycin blocks complex $\mathrm{V}$, resulting in Leak respiration, which determines proton leak across the inner mitochondrial membrane independently of complex V. Stepwise addition of $1 \mathrm{mM}$ carbonyl cyanide $\mathrm{p}$ trifluoro-methoxyphenyl hydrazone (FCCP) reveals the electron transport system (ETS) capacity, which determines the maximum respiratory capacity under infinite energy demand. Lastly, $5 \mu \mathrm{M}$ antimycin $\mathrm{A}$ was added to determine background respiration, which is subtracted from the other respiratory states. 
Respirometry and parallel ROS production using Amplex UltraRed were performed as described previously (26). Briefly, air calibration was performed using the same settings as for intact cells, but in Mir05 respiratory buffer [0.5 mM EGTA, $3 \mathrm{mM} \mathrm{MgCl}$, $60 \mathrm{mM}$ lactobionic acid, $20 \mathrm{mM}$ taurine, $10 \mathrm{mM} \mathrm{KH}_{2} \mathrm{PO}_{4}$, 20 mM HEPES, 110 mM D-sucrose, and fatty acid-free BSA (1 g/l) and 5 mM DTPA]. After air calibration, the chambers were closed and $10 \mu \mathrm{M}$ Amplex Ultrared (10 mM stock in DMSO), $0.5 \mathrm{U} / \mathrm{ml}$ horseradish peroxidase $(500 \mathrm{U} / \mathrm{ml}$ stock in Mir05), and $5 \mathrm{U} / \mathrm{ml}$ superoxide dismutase $(5000 \mathrm{U} / \mathrm{ml}$ stock in Mir05) were added. Subsequently, ROS calibration was performed with 2 consecutive injection of $0.2 \mu \mathrm{M}$ hydrogen peroxide (Fluorescence-sensor Green, gain for sensor $=1000$, polarization voltage $=300 \mathrm{mV}$ ).

Cells were harvested and 2 million cells in $400 \mu$ Mir05 were added to each chamber. Cells were permeabilized by addition of $5 \mu \mathrm{g} / \mathrm{ml}$ digitonin. Addition of $5 \mathrm{mM}$ pyruvate, $2 \mathrm{mM}$ malate, $10 \mathrm{mM}$ glutamate and $5 \mathrm{mM}$ adenosine diphosphate (ADP) resulted in complex I-linked respiration. Further addition of $10 \mathrm{mM}$ succinate revealed OxPhos capacity, the maximal capacity of mitochondria to produce ATP upon substrate saturation. Addition of $1.25 \mu \mathrm{M}$ oligomycin resulted in Leak respiration and stepwise addition of FCCP resulted in ETS capacity. Addition of $2.5 \mu \mathrm{M}$ rotenone blocked complex I and revealed complex II-linked respiration. Lastly, $5 \mu \mathrm{M}$ antimycin A was added to determine background respiration, which is subtracted from the other respiratory states. ROS production at the different respiratory states were first corrected for background flux before addition of cells and calculated as ROS production/respiration normalized to control.

\section{Flow Cytometry}

For all flow cytometry experiments, cells were seeded $48 \mathrm{~h}$ prior in 775 cell culture flasks in their respective medium. The day of the experiment, cells were harvested, counted and for each condition 1 million alive cells were stained in Tyrodes buffer $\left(135 \mathrm{mM} \mathrm{NaCl}, 5 \mathrm{mM} \mathrm{KCl}, 1.8 \mathrm{mM} \mathrm{CaCl}_{2}, 20 \mathrm{mM} \mathrm{HEPES}, 5 \mathrm{mM}\right.$ glucose, $1 \mathrm{mM} \mathrm{MgCl}_{2}, \mathrm{pH}$ 7.4) with the respective dye. Dependent on the dye, cells were washed in Tyrodes buffer, 
divided into 2 technical replicates, and 10,000 cells were measured for each replicate in Tyrodes buffer on an LSR Fortessa (BD). Alive singlets were gated in the FSC/SSC scatter plot and the mean fluorescence of the respective channel was quantified, corrected for the mean fluorescence of an unstained cell sample in that channel. Mitochondrial localization of the mitochondrial dyes following the respective staining procedures was checked prior to flow cytometry experiments using confocal microscopy.

\section{Mitochondrial mass using Mitotracker}

For determination of mitochondrial mass, cells were stained with $100 \mathrm{nM}$ MitoTracker ${ }^{\mathrm{TM}}$ Deep Red FM or $100 \mathrm{nM}$ MitoTracker ${ }^{\mathrm{TM}}$ Red CMXRos for $30 \mathrm{~min}$ at $37^{\circ} \mathrm{C}$. The mean fluorescence was quantified in the APC channel (ex: 640 nm, em: 670/14 nm) for MitoTracker ${ }^{\mathrm{TM}}$ Deep Red FM or the mCherry channel (ex:561nm, em:610/20 nm) for MitoTracker ${ }^{\mathrm{TM}}$ Red CMXRos.

\section{Mitochondrial Membrane Potential using JC1}

For determination of mitochondrial membrane potential, cells were stained with $1 \mu \mathrm{g} / \mathrm{ml} \mathrm{JC}-1$ for $30 \mathrm{~min}$ at $37^{\circ} \mathrm{C}$. The mean fluorescence was quantified in the FITC channel (ex:488 nm, em:530/30 nm) and the PE channel (ex:561 nm, em:582/15 nm), and the ratio of PE/FITC was calculated as a surrogate for membrane potential. This was verified by measuring cells treated with $5 \mu \mathrm{M} \mathrm{FCCP} \mathrm{upon} \mathrm{staining,} \mathrm{which}$ showed a lower PE/FITC fluorescence ratio.

\section{Mitochondrial ROS}

For determination of mitochondrial ROS, cells were stained with $5 \mu \mathrm{M}$ MitoSOX ${ }^{\mathrm{TM}}$ Red for 15 min at $37^{\circ} \mathrm{C}$. The cells were spun down and resuspended in fresh Tyrodes buffer. The mean fluorescence was quantified in the PerCP channel (ex:488 nm, em:695/40 nm).

\section{Cytosolic ROS}


For determination of cytosolic ROS, cells were stained with $1 \mu \mathrm{M} \mathrm{H} 2 \mathrm{DCFDA}$ for 30 min at $37^{\circ} \mathrm{C}$. The mean fluorescence was quantified in the FITC channel (ex:488 nm, em:530/30 nm).

\section{Mitochondrial calcium}

For determination of mitochondrial calcium, cells were stained with $10 \mu \mathrm{M}$ Rhod-2 AM (1 mM stock in DMSO with potassium borohydride) $+0.02 \%$ pluronic acid for $30 \mathrm{~min}$ at room temperature. The cells were washed once and resuspended in fresh Tyrodes buffer. The mean fluorescence was quantified in the PE channel (ex:561 nm, em:582/15 nm).

\section{Cytosolic calcium}

For determination of cytosolic calcium, cells were stained with $1 \mu \mathrm{M}$ Fura $\operatorname{Red}^{\mathrm{TM}} \mathrm{AM}+0.02 \%$ pluronic acid for $30 \mathrm{~min}$ at $37^{\circ} \mathrm{C}$. The cells were spun down and resuspended in fresh Tyrodes buffer. The mean fluorescence was quantified in the BV650 channel (ex:405 nm, em:655/8 nm) and the PerCP channel (ex:488 nm, em:695/40 nm). The ratio of BV650/PerCP was calculated as a surrogate for membrane potential. In confocal microscopy, cells were excited at $405 \mathrm{~nm}$ and $488 \mathrm{~nm}$ and emission was detected between $500 \mathrm{~nm}$ to $758 \mathrm{~nm}$.

\section{Apoptosis}

For determination of apoptosis, cells were harvested, washed in DPBS and 0.5 million cells were stained in $200 \mu$ l annexin-binding buffer (10 mM HEPES, $140 \mathrm{mM} \mathrm{NaCl}_{2}, 2.5 \mathrm{mM} \mathrm{CaCl}_{2}$, $\mathrm{pH}$ 7.4) supplemented with either $10 \mu$ l Annexin V Pacific Blue ${ }^{\mathrm{TM}}$ or $10 \mu$ I Annexin V FITC ${ }^{\mathrm{TM}}$ for $15 \mathrm{~min}$ at room temperature. After staining, $800 \mu \mathrm{l}$ annexin-binding buffer was added and the cells were measured using flow cytometry. The mean fluorescence was quantified in the Pacific Blue channel (ex:405 nm, em:450/50 nm) or the FITC channel (ex:488 nm, em:530/30 nm), respectively. No live-cell gate was applied and percentage of positive cells was quantified and normalized to control. 


\section{Mitochondrial permeability transition pore (mPTP) opening}

Mitochondrial permeability transition pore (MPTP) opening was measured using the calein-cobalt method as described (27). Briefly, cells were stained in Kreb's Ringer buffer (KRB) (135 mM NaCl, 5 mM KCl, 0.4 $\mathrm{mM} \mathrm{KH}_{2} \mathrm{PO}_{4}, 1 \mathrm{mM} \mathrm{MgSO}_{4}, 20$ mM HEPES, $5.5 \mathrm{mM}$ glucose, pH 7.4) supplemented with $1 \mu \mathrm{M}$ calcein, 200 nM MitoTracker ${ }^{\mathrm{TM}}$ Red CMXRos, $2 \mathrm{mM} \mathrm{CoCl}_{2}$ and $200 \mu \mathrm{M}$ sulfinpyrazone for $15 \mathrm{~min}$ at $37^{\circ} \mathrm{C}$. Cells were washed once in modified $\mathrm{KRB}\left(\mathrm{KBR}+1 \mathrm{mM} \mathrm{CaCl}_{2}\right)$, split and resuspended in modified $\mathrm{KRB}$ with and without $1 \mu \mathrm{M}$ ionomycin (positive control), and measured using flow cytometry. The mean fluorescence was quantified in the FITC channel (ex:488 nm, em:530/30 nm) for calcein and the mCherry channel (ex:561 $\mathrm{nm}$, em:610/20 nm) for MitoTracker ${ }^{\mathrm{TM}}$ Red CMXRos. mPTP opening was calculated as the mean calcein fluorescence of cells treated with ionomycin divided by the mean calcein fluorescence of untreated cells and then normalized to control cells.

\section{Confocal microscopy}

For all confocal microscopy experiments, 50,000 cybrids were seeded in each quadrant of 4-chambered $35 \mathrm{~mm}$ dishes with glass bottom (Cellview, 627870) in their standard culture medium $48 \mathrm{~h}$ prior to the measurement. On the day of the experiment, medium was exchanged for the respective staining solution and cells were imaged in Tyrodes buffer at $37^{\circ} \mathrm{C}$ and atmospheric $\mathrm{CO}_{2}$ after a 15 min equilibration in the microscope incubation chamber. For lymphoblasts, the 4-chambered $35 \mathrm{~mm}$ dishes with glass bottom were precoated with $2.5 \mu \mathrm{g} / \mathrm{cm}^{2}$ Cell-Tak $^{\mathrm{TM}}$ (Corning) for $30 \mathrm{~min}$ at $37^{\circ} \mathrm{C}$, rinsed with PBS twice and 500,000 cells were seeded into each quadrant in Tyrodes buffer immediately prior to the 15 min incubation in the microscope incubation chamber. Imaging was performed using a Zeiss LSM 710 in combination with the Zen 2012 software for image acquisition and analysis.

\section{Fluorescence lifetime imaging microscopy (FLIM) of NADH autofluorescence}


Fluorescence lifetime imaging of NADH was performed on a laser scanning microscope (Zeiss LSM 710) as described (28). Briefly, NADH was excited using two-photon excitation with a pulsed ( $80 \mathrm{MHz}, 100 \mathrm{fs}$ pulse width) titanium-sapphire laser at a power of $<5 \mathrm{~mW}$ on the sample. Time-correlated single photon counting (TCSPC) with the hybrid detector HPM-100-40 was performed. The detector was coupled to the NDD port of the LSM 710. FLIM images ( $512 \times 512$ pixel) were taken at a temporal resolution of 256 time channels within a pulse period of $12.5 \mathrm{~ns}$. Final settings: 60 sec collection time; $\approx 15 \mu$ sec pixel dwell time; $135 \times 135 \mu \mathrm{m}^{2}$ scanning area, Plan-Apochromat 63x/1.40 Oil DIC M27 lense, $730 \mathrm{~nm}$ excitation wavelength and 460/50 nm bandpass emission filter. Data were recorded using SPCM 9.8 and subsequently analyzed using SPCImage 8.0 assuming a biexponential decay. Final analysis settings: WLS fit method, lifetime components fixed to 400 ps and 2500 ps for free and protein-bound NADH, square binning of 2, peak threshold adapted to background, shift fixed at a pixel of clear NADH signal. The mean lifetime $\left(\tau_{\text {mean }}\right)$ was calculated and fitting of the calculated lifetime curve was confirmed by checking the mean $\chi^{2}$, which should be below 1.2. For subcellular analysis, nuclei were selected using region of interests (ROIs) with at least five nuclei analyzed per image. The mitochondria-rich regions were selected using the threshold function.

\section{RNA Sequencing and analysis}

For RNA sequencing, cybrids were seeded and cultured as for biochemical measurements, harvested and a cell pellet of 1.5 million cells was flash frozen and sent to Genewiz (Azenta Life Science, South Plainfield, NJ) for RNA sequencing using Poly-A-primers. Eighteen Human Tourett RNA-sequencing Fastq files, consisting of 6 controls and 12 mutant samples, were processed using the STAR alignment (29) tool and subsequently normalized using the RSEM (30) package based upon the hg38 reference genome (31) and the Gencode version 23 gene annotation (32).

Differential gene expression analysis was performed by comparing each gene in the control group vs the treatment group. The voom procedure (33) was used to normalize the RSEM generated expected counts 
followed by differential expression testing using R package limma (34) to obtain P-values and LogFC.

Specifically, a total of 58581 genes were tested for differential expression between the control and treatment samples. Pathway enrichment was performed on control vs treatment samples using Gene Set Enrichment Analysis (GSEA) $(35,36)$ version 4.1 .0 using a weighted scoring scheme and Hallmark and C2 CP genesets.

All input data and code can be found on github: https://github.com/komalsrathi/tourette-analysis

\section{Metabolomics}

For metabolomics, 1.5 million cybrids were seeded in $10 \mathrm{~cm}$ cell culture dishes and cultured using standard culture conditions for $48 \mathrm{~h}$. The day of the experiment, cells were transferred on ice into a cold room, medium was removed, cells were rinsed once using ice-cold PBS and liquid nitrogen was poured directly on the cells. The cell slush was scrapped of and transferred into a $1.5 \mathrm{ml}$ tube, that was flash frozen in liquid nitrogen and stored at $-80^{\circ} \mathrm{C}$ until metabolite preparation. A separate aliquot was taken for determination of protein concentration using BCA.

For untargeted LC/MS metabolomics, $50 \mu \mathrm{L}$ of each thawed cell pellet on ice was aliquoted for untargeted metabolomics, and a $5 \mu \mathrm{L}$ aliquot of each sample was pooled together and divided to make six $50 \mu \mathrm{L} \mathrm{QC}$ samples. Methanol (200 $\mu \mathrm{L}$ ) was added to each sample, vortexed vigorously for $10 \mathrm{sec}$, and centrifuged at $14000 \mathrm{rpm}$ for $10 \mathrm{~min}$ at $4{ }^{\circ} \mathrm{C}$. Then, $2 \times 100 \mu \mathrm{L}$ aliquots (one for reversed phase $\mathrm{C} 18 \mathrm{LC} / \mathrm{MS}$ and one for HILIC/MS) of the supernatant were placed into one of two 96 well plates and dried under nitrogen at 30 ${ }^{\circ} \mathrm{C}$. Dried samples were reconstituted in $200 \mu \mathrm{L}$ of HPLC mobile phases before LC/MS analysis on a Thermo Vanquish UHPLC/Orbitrap ID-X mass spectrometer. Compound Discoverer (Thermo Fisher Scientific) was used to generate PCA plots from the metabolite signals extracted from the raw data files, fold changes, $p$ values, heat maps, whisker plots, and perform a database search for metabolite identification. Pathway 
analysis was performed in MetaboAnalyst $5.0(37)$, using all metabolites that are significantly $(p<0.05)$ up or down in mutant versus control cybrids.

For targeted LC/MS metabolomics of organic acids and malonyl and acetyl CoA, aliquots (100 $\mu \mathrm{L})$ of frozen cell lysates were homogenized in equal volumes of acetonitrile/0.6 \% formic acid. Metabolites were extracted in cold aqueous/organic solvent mixtures according to validated, optimized protocols in our previously published studies $(38,39)$. These protocols use cold conditions and solvents to arrest cellular metabolism and maximize the stability and recovery of metabolites. Each class of metabolites was separated with a unique HPLC method to optimize their chromatographic resolution and sensitivity. Quantitation of metabolites in each assay module was achieved using multiple reaction monitoring of calibration solutions and study samples on an Agilent 1290 Infinity UHPLC/6495 triple quadrupole mass spectrometer (39). Raw data were processed using Mass Hunter quantitative analysis software (Agilent). Calibration curves ( $R 2=0.99$ or greater) were either fitted with a linear or a quadratic curve with a $1 / \mathrm{X}$ or 1/X2 weighting.

\section{Posttranslational modifications of histones}

Histone isolation from cybrids was performed as described previously (40). Briefly, cells were lysed in nuclear isolation buffer (NIB, $15 \mathrm{mM}$ Tris, $60 \mathrm{mM} \mathrm{KCl}, 15 \mathrm{mM} \mathrm{NaCl}, 5 \mathrm{mM} \mathrm{MgCl}$, $1 \mathrm{mM} \mathrm{CaCl}$, $250 \mathrm{mM}$ sucrose, $1 \mathrm{mM}$ DTT, $500 \mu \mathrm{M}$ AEBSF, $5 \mathrm{nM}$ microcystin, $10 \mathrm{mM}$ sodium butyrate) supplemented with $0.3 \%$ NP-40 for 5 min, nuclei were pelleted and washed three times in NIB. Histones were extracted in $0.2 \mathrm{M}$ sulfuric acid for $4 \mathrm{~h}$ and precipitated from the sulfuric acid using $20 \%$ TCA. Histone precipitate was rinsed using ice-cold acetone+0.1\% HCL, air-dried, resuspended in water and the concentration determined using BCA.

For histone mass spectrometry, primary amines and monomethyllysine residues of histones were derivatized using propionic anhydride in acetonitrile and ammonium hydroxide. Histones were dried, 
digested using trypsin, and then propionylation was performed again to derivatize the newly generated $\mathrm{N}$-termini. Samples were dried then desalted using in-house stagetips. Peptides were separated on an EASY-nLC 1000 using $0.1 \%$ formic acid in water as buffer A, $0.1 \%$ formic acid in acetonitrile as buffer B, and C18 as trap and analytical stationary phases (41). Data were acquired on a Thermo Q Exactive using DIA and processed in EpiProfile 2.1 (42).

\section{Acknowledgements}

We would like to thank Shiping Zhang for programming the mtDNA allele co-occurrence tool, enabling the analysis in Fig.S1c. We would further like to express our gratitude to Christopher Petucci at the Metabolomics Core at the University of Pennsylvania for performing and analyzing the global and targeted metabolomics and to the Flow Cytometry Core at CHOP for their training in flow cytometry. Lastly, we would like to thank Niagen ${ }^{\circledR}$ for providing the NR.

\section{Funding}

This work was supported by the German Research Foundation (SCHA 2182/1-1) to PM Schaefer and National Institutes of Health grants NS021328, MH108592, OD010944 plus U.S. Department of Defense grants W81XWH-16-1-0401 and W81XWH-21-1-0128 (PR202887.e002) awarded to DC Wallace.

\section{Author Contributions}

PMS, DCW designed the study; PMS, LSA, ML, JH, KJ, AB, TY, RM performed the experiments; PMS, KR, $\mathrm{KJ}, \mathrm{MJ}, \mathrm{BAG}$ analyzed the data; KK, CAF, DCW performed the clinical evaluation; PMS and DCW wrote the manuscript. All authors read and approved the manuscript.

\section{References}

1. Picard $M$, Wallace DC, Burelle $\mathrm{Y}$. The rise of mitochondria in medicine. Mitochondrion. 2016;30:105-16. 
2. Wallace DC. Mitochondrial genetic medicine. Nat Genet. 2018;50(12):1642-9.

3. Wallace DC, Singh G, Lott MT, Hodge JA, Schurr TG, Lezza AM, et al. Mitochondrial DNA mutation associated with Leber's hereditary optic neuropathy. Science. 1988;242(4884):1427-30.

4. Chalkia D, Singh LN, Leipzig J, Lvova M, Derbeneva O, Lakatos A, et al. Association Between Mitochondrial DNA Haplogroup Variation and Autism Spectrum Disorders. JAMA Psychiatry. 2017;74(11):1161-8.

5. Wallace DC. Mitochondrial DNA variation in human radiation and disease. Cell. 2015;163(1):33-8.

6. Yardeni T, Cristancho AG, McCoy AJ, Schaefer PM, McManus MJ, Marsh ED, et al. An mtDNA mutant mouse demonstrates that mitochondrial deficiency can result in autism endophenotypes. Proc Natl Acad Sci U S A. 2021;118(6).

7. Ercan-Sencicek AG, Stillman AA, Ghosh AK, Bilguvar K, O'Roak BJ, Mason CE, et al. L-histidine decarboxylase and Tourette's syndrome. N Engl J Med. 2010;362(20):1901-8.

8. Lott MT, Leipzig JN, Derbeneva O, Xie HM, Chalkia D, Sarmady M, et al. mtDNA Variation and Analysis Using Mitomap and Mitomaster. Curr Protoc Bioinformatics. 2013;44:1.23.1-6.

9. Huang H, Li Y, Liang J, Finkelman FD. Molecular Regulation of Histamine Synthesis. Front Immunol. 2018;9:1392.

10. Kotlikoff MI, Murray RK, Reynolds EE. Histamine-induced calcium release and phorbol antagonism in cultured airway smooth muscle cells. Am J Physiol. 1987;253(4 Pt 1):C561-6.

11. Sharpley MS, Marciniak C, Eckel-Mahan K, McManus M, Crimi M, Waymire K, et al. Heteroplasmy of mouse mtDNA is genetically unstable and results in altered behavior and cognition. Cell. 2012;151(2):333-43.

12. Latorre-Pellicer A, Moreno-Loshuertos R, Lechuga-Vieco AV, Sánchez-Cabo F, Torroja C, AcínPérez R, et al. Mitochondrial and nuclear DNA matching shapes metabolism and healthy ageing. Nature. 2016;535(7613):561-5.

13. Latorre-Pellicer A, Lechuga-Vieco AV, Johnston IG, Hämäläinen RH, Pellico J, Justo-Méndez R, et al. Regulation of Mother-to-Offspring Transmission of mtDNA Heteroplasmy. Cell Metab. 2019;30(6):1120-30.e5.

14. Lechuga-Vieco AV, Latorre-Pellicer A, Johnston IG, Prota G, Gileadi U, Justo-Méndez R, et al. Cell identity and nucleo-mitochondrial genetic context modulate OXPHOS performance and determine somatic heteroplasmy dynamics. Sci Adv. 2020;6(31):eaba5345.

15. Leclerc $C$, Néant I, Moreau M. The calcium: an early signal that initiates the formation of the nervous system during embryogenesis. Front Mol Neurosci. 2012;5:3.

16. Polyak E, Ostrovsky J, Peng M, Dingley SD, Tsukikawa M, Kwon YJ, et al. N-acetylcysteine and vitamin $\mathrm{E}$ rescue animal longevity and cellular oxidative stress in pre-clinical models of mitochondrial complex I disease. Mol Genet Metab. 2018;123(4):449-62.

17. Bowen JM, Sobey GJ, Burrows NP, Colombi M, Lavallee ME, Malfait F, et al. Ehlers-Danlos syndrome, classical type. Am J Med Genet C Semin Med Genet. 2017;175(1):27-39.

18. Irwin WA, Bergamin N, Sabatelli P, Reggiani C, Megighian A, Merlini L, et al. Mitochondrial dysfunction and apoptosis in myopathic mice with collagen VI deficiency. Nat Genet. 2003;35(4):367-71.

19. Palma E, Tiepolo T, Angelin A, Sabatelli P, Maraldi NM, Basso E, et al. Genetic ablation of cyclophilin $D$ rescues mitochondrial defects and prevents muscle apoptosis in collagen VI myopathic mice. Hum Mol Genet. 2009;18(11):2024-31.

20. Hernández-Muñoz R, Díaz-Muñoz M, Chagoya de Sánchez V. Possible role of cell redox state on collagen metabolism in carbon tetrachloride-induced cirrhosis as evidenced by adenosine administration to rats. Biochim Biophys Acta. 1994;1200(2):93-9.

21. Baldan LC, Williams KA, Gallezot JD, Pogorelov V, Rapanelli M, Crowley $M$, et al. Histidine decarboxylase deficiency causes tourette syndrome: parallel findings in humans and mice. Neuron. 2014;81(1):77-90. 
22. Abdurakhmanova S, Chary K, Kettunen M, Sierra A, Panula P. Behavioral and stereological characterization of Hdc KO mice: Relation to Tourette syndrome. J Comp Neurol. 2017;525(16):3476-87.

23. Pei L, Wallace DC. Mitochondrial Etiology of Neuropsychiatric Disorders. Biol Psychiatry. 2018;83(9):722-30.

24. Trounce IA, Kim YL, Jun AS, Wallace DC. Assessment of mitochondrial oxidative phosphorylation in patient muscle biopsies, lymphoblasts, and transmitochondrial cell lines. Methods Enzymol. 1996;264:484-509.

25. Schaefer PM, von Einem B, Walther P, Calzia E, von Arnim CA. Metabolic Characterization of Intact Cells Reveals Intracellular Amyloid Beta but Not Its Precursor Protein to Reduce Mitochondrial Respiration. PLoS One. 2016;11(12):e0168157.

26. Komlódi T, Sobotka O, Krumschnabel G, Bezuidenhout N, Hiller E, Doerrier C, et al. Comparison of Mitochondrial Incubation Media for Measurement of Respiration and Hydrogen Peroxide Production. Methods Mol Biol. 2018;1782:137-55.

27. Bonora M, Morganti C, Morciano G, Giorgi C, Wieckowski MR, Pinton P. Comprehensive analysis of mitochondrial permeability transition pore activity in living cells using fluorescence-imaging-based techniques. Nat Protoc. 2016;11(6):1067-80.

28. Schaefer PM, Hilpert D, Niederschweiberer M, Neuhauser L, Kalinina S, Calzia E, et al. Mitochondrial matrix $\mathrm{pH}$ as a decisive factor in neurometabolic imaging. Neurophotonics. 2017;4(4):045004.

29. Dobin A, Davis CA, Schlesinger F, Drenkow J, Zaleski C, Jha S, et al. STAR: ultrafast universal RNAseq aligner. Bioinformatics. 2013;29(1):15-21.

30. Li B, Dewey CN. RSEM: accurate transcript quantification from RNA-Seq data with or without a reference genome. BMC Bioinformatics. 2011;12:323.

31. Karolchik D, Baertsch R, Diekhans M, Furey TS, Hinrichs A, Lu YT, et al. The UCSC Genome Browser Database. Nucleic Acids Res. 2003;31(1):51-4.

32. Harrow J, Frankish A, Gonzalez JM, Tapanari E, Diekhans M, Kokocinski F, et al. GENCODE: the reference human genome annotation for The ENCODE Project. Genome Res. 2012;22(9):1760-74.

33. Law CW, Chen Y, Shi W, Smyth GK. voom: Precision weights unlock linear model analysis tools for RNA-seq read counts. Genome Biol. 2014;15(2):R29.

34. Ritchie ME, Phipson B, Wu D, Hu Y, Law CW, Shi W, et al. limma powers differential expression analyses for RNA-sequencing and microarray studies. Nucleic Acids Res. 2015;43(7):e47.

35. Subramanian A, Tamayo P, Mootha VK, Mukherjee S, Ebert BL, Gillette MA, et al. Gene set enrichment analysis: a knowledge-based approach for interpreting genome-wide expression profiles. Proc Natl Acad Sci U S A. 2005;102(43):15545-50.

36. Mootha VK, Lindgren CM, Eriksson KF, Subramanian A, Sihag S, Lehar J, et al. PGC-1alpharesponsive genes involved in oxidative phosphorylation are coordinately downregulated in human diabetes. Nat Genet. 2003;34(3):267-73.

37. Chong J, Soufan O, Li C, Caraus I, Li S, Bourque G, et al. MetaboAnalyst 4.0: towards more transparent and integrative metabolomics analysis. Nucleic Acids Res. 2018;46(W1):W486-W94.

38. Lanfear DE, Gibbs JJ, Li J, She R, Petucci C, Culver JA, et al. Targeted Metabolomic Profiling of Plasma and Survival in Heart Failure Patients. JACC Heart Fail. 2017;5(11):823-32.

39. Gardell SJ, Zhang X, Kapoor N, Petucci C, Coen PM. Metabolomics Analyses of Muscle Atrophy Induced by Hind Limb Unloading. Methods Mol Biol. 2019;1996:297-309.

40. Kopinski PK, Janssen KA, Schaefer PM, Trefely S, Perry CE, Potluri P, et al. Regulation of nuclear epigenome by mitochondrial DNA heteroplasmy. Proc Natl Acad Sci U S A. 2019;116(32):16028-35.

41. Karch KR, Sidoli S, Garcia BA. Identification and Quantification of Histone PTMs Using HighResolution Mass Spectrometry. Methods Enzymol. 2016;574:3-29. 
42. Yuan ZF, Sidoli S, Marchione DM, Simithy J, Janssen KA, Szurgot MR, et al. EpiProfile 2.0: A Computational Platform for Processing Epi-Proteomics Mass Spectrometry Data. J Proteome Res. 2018;17(7):2533-41.

\section{Figure Legends}

\section{Fig.1 ND5 m.13708G>A-H7 mtDNA reduces bioenergetic function}

a) Two generation pedigree with squares indicating males, circles females and triangles miscarriages. Black color indicates Tourette syndrome phenotype, grey-shaded indicates connective tissue phenotype. Chromosomes indicate the genotype for chromosome 15, with the father and all children being heterozygous for the W317X HDC variant. Red mitochondria indicate maternal mitochondria harboring the ND5 m.13708G>A variant on haplogroup H7 background. b) Respirometry of intact transmitochondrial cybrids harboring control (black) or patient (mutant, red) mitochondria (control: $n=19$, mutant $n=31$, technical duplicates, Mann Whitney test). c) Mitochondrial membrane potential in control and mutant cybrids measured as red/green fluorescence of JC- 1 quantified by flow cytometry normalized to control ( $n=12$, technical duplicates, paired t-test). d) Fluorescence lifetime imaging microscopy (FLIM) of mitochondrial NADH in control and mutant cybrids. False-color coding of NADH fluorescent lifetime with blue indicating a longer lifetime (more oxidized $N A D^{+} / N A D H$ ) and red indicating a shorter lifetime (more reduced $\mathrm{NAD}^{+} / \mathrm{NADH}$ ). Grey indicates non-mitochondrial autofluorescence. Quantification of the mean mitochondrial NADH lifetime ( $n=5$ with 5 image sections and >25 cells/independent experiment, unpaired t-test). e) Cytosolic and mitochondrial ROS levels in control and mutant cybrids quantified as the fluorescence intensity of DCDFA (cytosol, $n=11$ in technical duplicates, paired t-test) or Mitosox (mitochondrial, $n=26$ in technical duplicates, paired t-test) normalized to control. f) Doubling time of control and mutant cybrids ( $n=20$, Wilcoxon signed rank test). Error bars display $95 \%$ confidence intervals (CI). Significances are indicated by stars with ${ }^{*}=p<0.05, * *=p<0.01, * * *=p<0.001$.

\section{Fig.2 ND5 m.13708G>A-H7 mtDNA profoundly alters metabolite and expression profile}

a) Cluster analysis from GSEA of RNASeq in mutant versus control cybrids using Cytoscape. Every node represents a significant pathway with blue indicating upregulation and red downregulation of the pathway in variant versus control. b) Fluorescence lifetime imaging microscopy (FLIM) of nuclear NADH in control and mutant cybrids. False-color coding of NADH lifetime with blue indicating a longer NADH fluorescent lifetime and a more oxidized NAD+/NADH redox ratio and red indicating a shorter NADH fluorescent lifetime or more reduced redox ratio. Grey indicates non-nuclear autofluorescence. Quantification of the mean nuclear NADH lifetime revealed a more reduced redox ratio in mutant cybrids ( $n=5$ with 5 image sections and $>25$ nuclei/independent experiment, unpaired t-test). c) Metabolic pathway analysis of global untargeted metabolomics using significantly $(p<0.05)$ altered metabolites between mutant and control cybrids. Size of the dots indicates pathway impact ( $x$-axis) and color (white-yellow - red) indicates p-value with red corresponding to higher significance. d) Selected histone modifications of histone 3 in control and mutant cybrids displayed as peptide abundance normalized to control. ( $n=6,12$, unpaired ttest). 
Fig.3 Calcium homeostasis links paternal HDC variant with the ND5 m.13708G >A-H7 mtDNA

a) Cytosolic and mitochondrial calcium levels in control and mutant cybrids normalized to control measured by confocal microscopy or flow cytometry using Fura Red (cytosol, $n=6$ microscopy, $n=7$ flow cytometry, paired t-test) or Rhod-2 (mitochondria, $\mathrm{n}=13$ flow cytometry, paired t-test). b) Cytosolic calcium levels in mutant and control cybrids in response to a $100 \mu \mathrm{M}$ histamine stimulus, normalized to calcium levels pre-addition (time point 0 ). Measured using Fura Red and confocal microscopy ( $n=7$, paired t-test). c/d) Electron transport system capacity (c) and mitochondrial ROS levels (d) of control and mutant cybrids with and without pretreatment with $100 \mu \mathrm{M}$ histamine for $24 \mathrm{~h}$. Significances between mutant and control $(*)$ and between treatments (\#) were calculated using Kruskal Wallis test ( $n=19,6,31,12$, technical duplicates) for respirometry and One-Way ANOVA ( $n=26,12,26,12$, technical duplicates) for ROS. e) Diagram visualizing the interaction between a pathogenic variant in the Histidine Decarboxylase and the ND5 m.13708G $>\mathrm{A}-\mathrm{H} 7 \mathrm{mtDNA}$. Blue arrows indicate an increase and red arrows a decrease in the mutant cybrids. The ND5 m.13708G>A-H7 mtDNA reduces respiration and one carbon metabolism, resulting in epigenetic and transcriptional changes that include an upregulation of Histamine 1 receptor (HRH1), Phospholipase C (PLCG1), and IP3 receptor (ITPR3). Upon histamine, this leads to increased calcium release from the ER, mitochondrial calcium overload, mitochondrial permeability transition pore (mPTP) opening, and apoptosis. The HDC variant reduces the formation of histamine from histidine, thereby preventing histamine toxicity. Created with BioRender.com.

\section{Fig.4 Patient lymphoblasts show increased ROS levels and a switch from complex I to complex II respiration}

Patient lymphoblastoid cells from the mother (12) and 4 offspring (II5-II8) are compared to 3 haplogroupmatched controls. a/e) Electron transport system capacity in intact control and patient lymphoblastoid cells $(n=14)$. b/f) Complex $1 /$ complex II respiration in permeabilized lymphoblastoid cells $(n=$ $11,8,10,13,13,9,12,9) \mathrm{c} / \mathrm{g}$ ) Cytosolic ROS levels in control and patient lymphoblastoid cells quantified as the fluorescence intensity of DCDFA normalized to control ( $n=16,16,15,15,14,16,15,16)$. $d / h$ ) Mitochondrial mass of control and patient lymphoblastoid cells quantified as fluorescence intensity of Mitotracker CMX ROS or Mitotracker Deep Red in flow cytometry normalized to control ( $n=11$ ). Significance for patient versus control (a-d) was calculated using unpaired t-test and significances for individual patients versus individual controls (e-h) were calculated using One-way ANOVA or Kruskal Wallis test. Significances relative to control are indicated by * for C1, \# for C2, and $\diamond$ for C3.

\section{Fig.S1 Mitochondrial haplogroup J defining variant ND5 m.13708G>A on haplogroup H7 background}

a) Mitochondrial variants found in the proband (mother, 12) and a closely matched control, indicating in which region/gene of the mtDNA the variant is localized, the nucleotide change, the associated amino acid change, and the frequency of the variant on Mitomap (as of 05/04/2021). Variants that result in an amino-acid change are marked in red, frequencies of the variants are colored white to red with red indicating rarer variants. b) Frequencies of $13708 \mathrm{~A}$ in different mtDNA haplogroups colored white to red with red indicating a lower frequency. Counts display the number of mtDNAs of the respective haplogroup 
on Mitomap (as of 05/04/2021). c) Normalized co-occurrence of mtDNA variants with values < 1 (red) indicating lower-than-expected co-occurrence within the population and values $>1$ (blue) a higher-thanexpected co-occurrence (Mitomap, as of 05/04/2021). Co-occurrence is displayed for all proband mtDNA variants and variants between mitochondrial haplogroup $\mathrm{H}$ and $\mathrm{J}$ that result in an amino acid change.

\section{Fig.S2 ND5 m.13708G>A-H7 mtDNA reduces bioenergetic function}

a) Diagram displaying creation of $143 \mathrm{~B}\left(\mathrm{TK}^{-}\right)$transmitochondrial cybrids. Lymphoblasts from control (grey) and proband (Fig. 1a, 12) (red) were enucleated and the resulting cytoplasts fused with $\mathrm{p}^{0} 143 \mathrm{~B}\left(\mathrm{TK}^{-}\right)$cells that are depleted of their mtDNA. After selection, the resulting cybrids contain the $143 \mathrm{~B}\left(\mathrm{TK}^{-}\right)$nuclear background with either the patient (mutant) or control mitochondria, allowing the deciphering of the influence of the mtDNA independent of nuclear genes. b) Respiratory control ratios of high-resolution respirometry in intact cybrids calculated from data in Fig.1b (Routine/ETS: fraction of maximal respiratory capacity used at baseline; Leak/ETS: fraction of maximal respiratory capacity that is not used for ATP production; Leak/Routine: fraction of baseline respiration that is not used for ATP production) (control: $n$ $=19$, mutant $n=31$, each in technical duplicates, Mann Whitney test). $\mathbf{c}$ ) Mitochondrial mass of control and mutant cybrids quantified as fluorescence intensity of Mitotracker CMX ROS in flow cytometry normalized to control ( $n=6$, technical triplicates, paired t-test). d) High resolution respirometry in permeabilized cybrids ( $n=7$, technical duplicates, Wilcoxon signed rank test) e) ROS production in permeabilized cybrids quantified as the increase in amplex red fluorescence at different respiratory states normalized to control ( $n=6$, technical duplicates, One-sample t-test). f) Apoptosis in cybrids quantified as the fluorescence intensity of the cells after Annexin $V$ staining using flow cytometry $(n=6$, technical duplicates, paired t-test).

\section{Fig.S3 Mitochondrial haplogroup J shows similar bioenergetics to haplogroup H}

a) Doubling time of haplogroup $\mathrm{H}$ and $\mathrm{J}$ cybrids in low glucose media ( $\mathrm{n}=17$, Mann Whitney test). b) High resolution respirometry of intact haplogroup $H$ (black) or J (grey) cybrids ( $n=8$, each in technical duplicates, Mann Whitney test). c) Mitochondrial mass of haplogroup $\mathrm{H}$ and $\mathrm{J}$ cybrids quantified as fluorescence intensity of Mitotracker Deep Red in flow cytometry normalized to haplogroup $\mathrm{H}(\mathrm{n}=6$, technical duplicates, paired t-test). d) Mitochondrial membrane potential of haplogroup $\mathrm{H}$ and $\mathrm{J}$ cybrids measured as the ratio of red to green fluorescence of JC-1 quantified by flow cytometry normalized to haplogroup $\mathrm{H}(\mathrm{n}=7$, technical duplicates, paired t-test). e) Cytosolic and Mitochondrial ROS levels of haplogroup $\mathrm{H}$ and $\mathrm{J}$ cybrids quantified as the fluorescence intensity of DCDFA (cytosol, $\mathrm{n}=3$ in technical duplicates, paired t-test) or Mitosox (mitochondrial, $n=3$ in technical duplicates, paired t-test) normalized to haplogroup H. f) Cytosolic and Mitochondrial calcium levels of haplogroup $\mathrm{H}$ and $\mathrm{J}$ cybrids quantified as the fluorescence intensity of Fura Red (cytosol, $n=7$ in technical duplicates, paired t-test) or Rhod-2 (mitochondrial, $\mathrm{n}=5$ in technical duplicates, paired t-test) normalized to haplogroup $\mathrm{H}$.

Fig.S4 ND5 m.13708G>A-H7 mtDNA alters nicotinamide, TCA cycle, and one carbon metabolism 
a) Differential gene expression between mutant and control cybrids for the KEGG Nicotinate and Nicotinamide Metabolism pathway. Log fold change (LogFC) is colored blue - white - red with blue indicating upregulation and red indicating downregulation of a gene in mutant cybrids compared to control. Adjusted (adj.) p-values are colored white - yellow with yellow indicating stronger significance. b) Relative nucleotide levels in mutant cybrids normalized to control ( $n=6 / 12$ for control/mutant, unpaired t-test) detected in global metabolomics. c) Targeted metabolomics of TCA cycle intermediates in mutant cybrids normalized to control ( $n=6$ for control, $n=12$ for mutant, One-sample t-test). d) Differential gene expression between mutant and control cybrids for the KEGG One Carbon Pool by Folate pathway. Log fold change (LogFC) is colored blue - white - red with blue indicating upregulation and red indicating downregulation of a gene in mutant cybrids compared to control. Adjusted (adj.) p-values are colored white - yellow with yellow indicating stronger significance. e) Targeted metabolomics of one carbon metabolism in mutant cybrids normalized to respective control ( $n=6$ for control, $n=12$ for mutant, One-sample t-test).

\section{Fig.S5 ND5 m.13708G>A-H7 mtDNA results in histone hypomethylation}

a/b) Upregulated (a) and downregulated (b) post-translational modifications (PTMs) from histones H3 and $\mathrm{H} 4$ in mutant compared to control cybrids measured using mass spectrometry. The ratio indicates the fraction of the peptide with the respective modification, and the color-coding the type of modification present on the peptide, with blue being unmodified, red being methylation, yellow acetylation and orange methylation and acetylation. c) Single peptide modifications independent of the originating histone in mutant compared to control cybrids. Significant PTMs are colored according to their modification. Significance versus control was calculated for all PTMs using unpaired t-test $(n=6)$.

\section{Fig.S6 ND5 m.13708G>A-H7 mtDNA alters cholesterol, ketone and collagen metabolism}

$\mathrm{a} / \mathrm{b} / \mathrm{c}$ ) Differential gene expression between mutant and control cybrids for the Reactome cholesterol biosynthesis (a), the Reactome Ketone Body Metabolism (b) pathway and the Reactome collagen formation pathway (c). Log fold change (LogFC) is colored blue - white - red with blue indicating upregulation and red indicating downregulation of a gene in mutant cybrids compared to control. Adjusted (adj.) p-values are colored white - yellow with yellow indicating stronger significance. d) Acylcarnitines detected in the global metabolomics screen in mutant cybrids normalized to control $(n=6$ for control, $n=12$ for mutant, unpaired t-test).

\section{Fig.S7 ND5 m.13708G>A-H7 mtDNA reduces mitochondrial calcium uptake and increases histamine sensitivity}

a) Differential gene expression between mutant and control cybrids for the Reactome mitochondrial calcium ion transport pathway. Log fold change ( $\operatorname{LogFC}$ ) is colored blue - white - red with blue indicating upregulation and red indicating downregulation of a gene in mutant cybrids compared to control. Adjusted (adj.) p-values are colored white - yellow with yellow indicating stronger significance. b) Mitochondrial calcium levels in mutant and control cybrids 2 min after a $100 \mu \mathrm{M}$ Histamine stimulus, 
normalized to calcium levels pre-histamine. Measured using Rhod-2 and flow cytometry ( $n=6$, each in technical duplicates, paired t-test). c) Cytosolic calcium levels in variant and control cybrids in response to a $100 \mu \mathrm{M}$ histamine stimulus after pretreatment with MCU-inhibitor (10 $\mu \mathrm{M}$ KB-R7943), normalized to calcium levels pre-histamine (time point 0 ). Measured using Fura Red and confocal microscopy ( $n=9$, paired t-test). d) Cytosolic calcium levels in mutant and control cybrids in response to a $2 \mu \mathrm{M}$ thapsigargin stimulus, normalized to calcium levels pre-thapsigargin (time point 0). Measured using Fura Red and confocal microscopy ( $n=6$, paired t-test). e) Differential gene expression between mutant and control cybrids for histamine 1 receptor (HRH1), phospholipase C (PLCG1) and IP3 receptor (ITPR3) and endoplasmatic reticulum protein 44 (ERP44), a negative regulator of IP3 receptor-mediated calcium release ( $n=6$ for control, $n=12$ for mutant, unpaired $t$-test).

Fig.S8 Histamine toxicity in ND5 m.13708G>A-H7 mtDNA cybrids is mediated via Histamine 1 receptormediated calcium release and $\mathrm{mPTP}$ opening

a) Routine respiration of intact control and mutant cybrids with and without pretreatment with $100 \mu \mathrm{M}$ histamine for $24 \mathrm{~h}$ measured using high-resolution respirometry $(n=19,6,31,12$ from left to right, each in technical duplicates, Kruskal Wallis test). b) mPTP opening in control and mutant cybrids with and without pretreatment with $100 \mu \mathrm{M}$ histamine for $24 \mathrm{~h}$ measured cobalt-calcein quenching in flow cytometry ( $n=6$, each in technical duplicates, One-Way ANOVA). c/d) Routine respiration (c) and electron transport system capacity (d) of intact pyrilamine-treated $(100 \mu \mathrm{M})$ control and mutant cybrids with and without treatment with $100 \mu \mathrm{M}$ histamine for $24 \mathrm{~h}$ measured using high-resolution respirometry ( $\mathrm{n}=8$, each in technical duplicates, Kruskal Wallis test). e) Mitochondrial ROS levels of pyrilamine-treated control and variant cybrids with and without treatment with $100 \mu \mathrm{M}$ histamine for $24 \mathrm{~h}$ quantified as the fluorescence intensity of Mitosox normalized to untreated control ( $n=7$, each in technical duplicates, One-way ANOVA). Significances between mutant and control are indicated by * and between treatment and control are indicated by \#.

\section{Fig.S9 Histamine 1 receptor inhibition reduced mitochondrial function but alleviates the negative effect of the ND5 m.13708G >A-H7 mtDNA}

Comparison of control and mutant cybrids untreated or cultured with $100 \mu \mathrm{M}$ pyrilamine added to the medium. a) Doubling time ( $n=20$, Kruskal Wallis test). b) Electron transport system capacity of intact cells ( $n=19,31,8,8$ from left to right, each in technical duplicates, One-Way ANOVA). c/d) Cytosolic (c) and mitochondrial (d) ROS levels quantified as the fluorescence intensity of DCDFA (cytosol, $n=11,11,6,6$ from left to right, in technical duplicates, One-way ANOVA) or Mitosox (mitochondrial, $n=26,26,6,6$ from left to right, in technical duplicates, One-way ANOVA) normalized to untreated control. e) Mitochondrial membrane potential measured as the ratio of red to green fluorescence of JC-1 quantified by flow cytometry normalized to untreated control $(n=12,12,6,6$ from left to right, each in technical duplicates, One-way ANOVA). f/g) Cytosolic (f) and mitochondrial (g) calcium levels measured by flow cytometry using Fura Red (cytosol, $n=13,13,7,7$ from left to right, each in technical duplicates, KruskalWallis test) or Rhod-2 (mitochondria, $\mathrm{n}=13,13,10,10$, each in technical duplicates, Kruskal-Wallis test) normalized to untreated control. h) Apoptosis quantified as the fluorescence intensity of the cells after Annexin $V$ staining using flow cytometry ( $n=6$, technical duplicates, Repeated measures ANOVA). 
Significances between mutant and control are indicated by ${ }^{*}$ and between treatment and control are indicated by \#.

Fig.S10 NR and NAC can partially rescue the mitochondrial defect in the ND5 m.13708G $>$ A-H7 mtDNA cybrids

Comparison of control and mutant cybrids untreated or cultured with either $300 \mu \mathrm{M}$ nicotinamide riboside or $1 \mathrm{mM} \mathrm{N}$-acetylcysteine added to the medium. a) Doubling time ( $n=20$, Kruskal Wallis test). b) Electron transport system capacity of intact cells $(n=19,31,8,8,8,8$ from left to right, each in technical duplicates, One-Way ANOVA). c) Cytosolic ROS levels quantified as the fluorescence intensity of DCDFA (cytosol, $n=11,11,6,6,6,6$ from left to right, in technical duplicates, One-way ANOVA) normalized to untreated control. d) Mitochondrial membrane potential measured as the ratio of red to green fluorescence of JC-1 quantified by flow cytometry normalized to untreated control ( $n=12,12,6,6,6,6$ from left to right, each in technical duplicates, One-way ANOVA). e/f) Cytosolic (e) and mitochondrial (f) calcium levels measured by flow cytometry using Fura Red (cytosol, $n=13,13,7,7$ from left to right, each in technical duplicates, Kruskal-Wallis test) or Rhod-2 (mitochondria, $n=13,13,10,10$, each in technical duplicates, Kruskal-Wallis test) normalized to untreated control. Significances between mutant and control are indicated by * and between treatment and control are indicated by \#. 
Fig.1 ND5 m.13708G>A-H7 mtDNA reduces bioenergetic function

a)

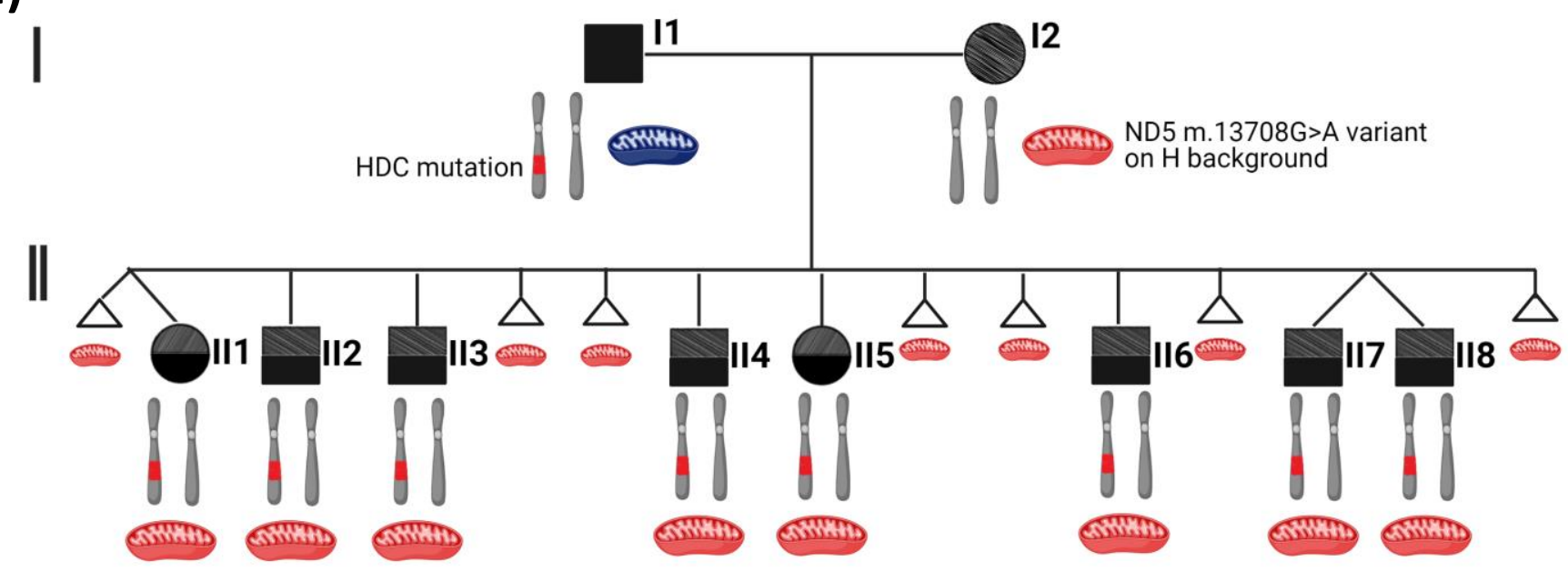

b)
d)

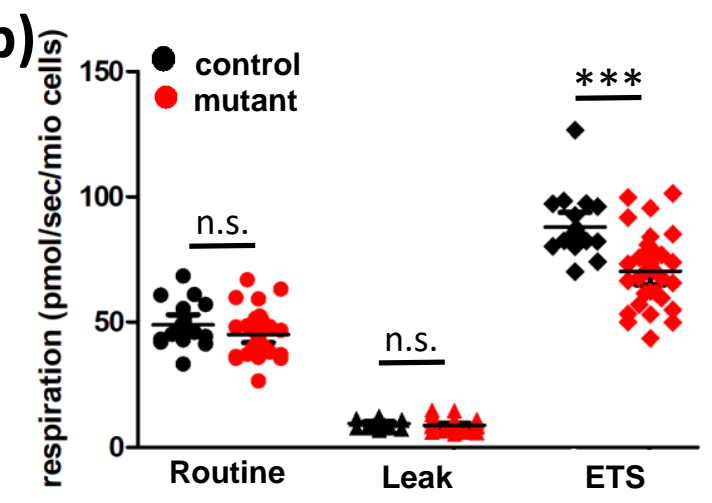

c)

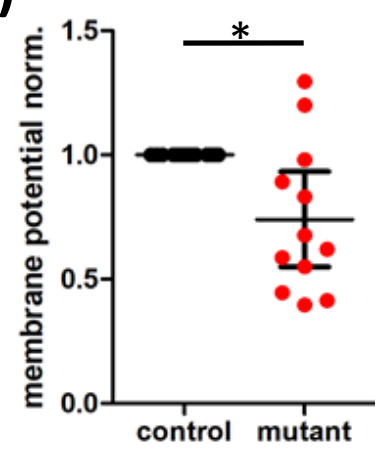

e)

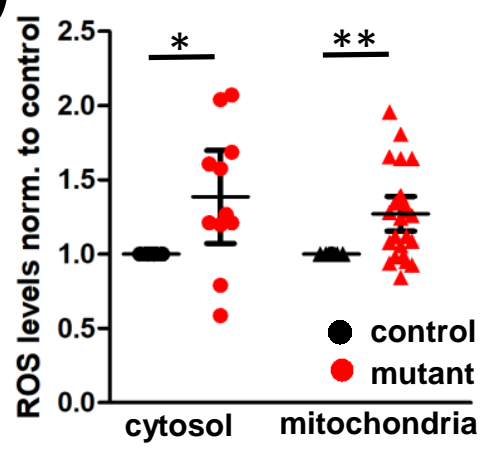

f)

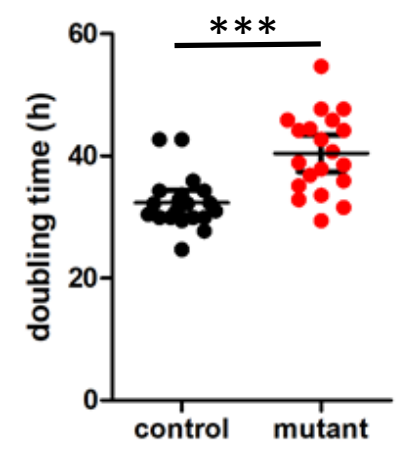




\section{Fig.2 ND5 m.13708G>A-H7 mtDNA profoundly alters metabolite and expression profile}

a)

\section{RNA Sequencing}

Histone

RNA metabolism modifications
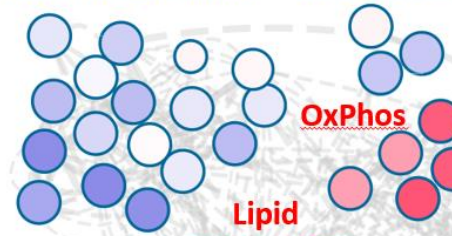

Oxphos

Lipid

Cholesterol metabolism homeostasis
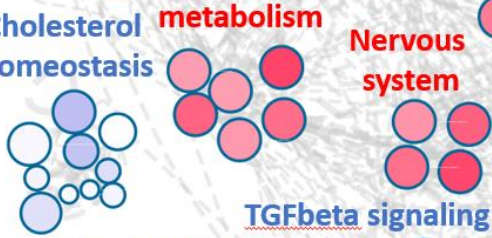

TGFbeta signaling
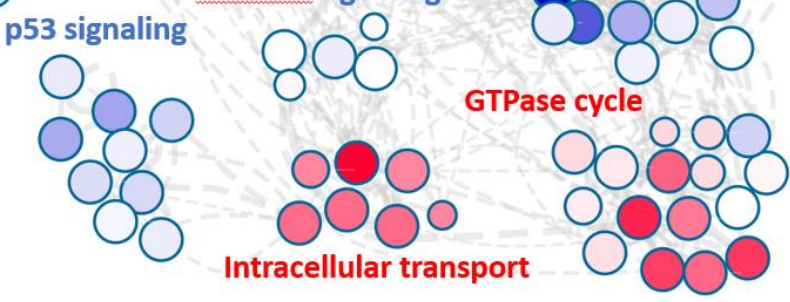

b)
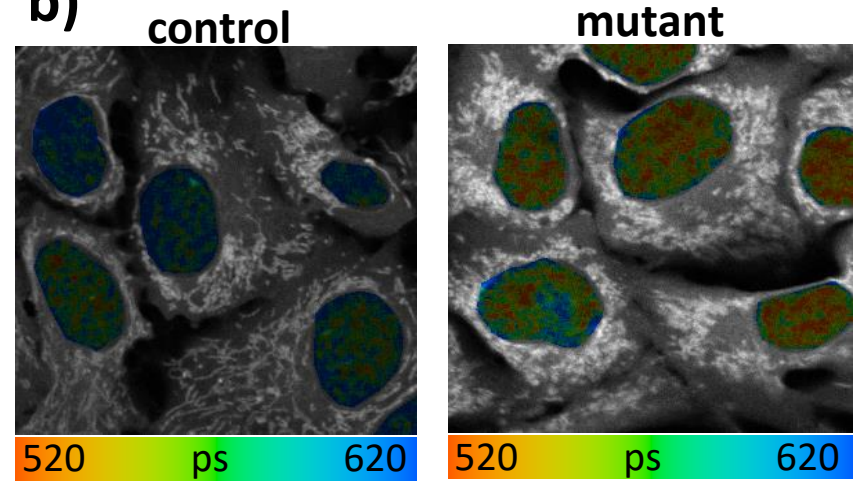

c)

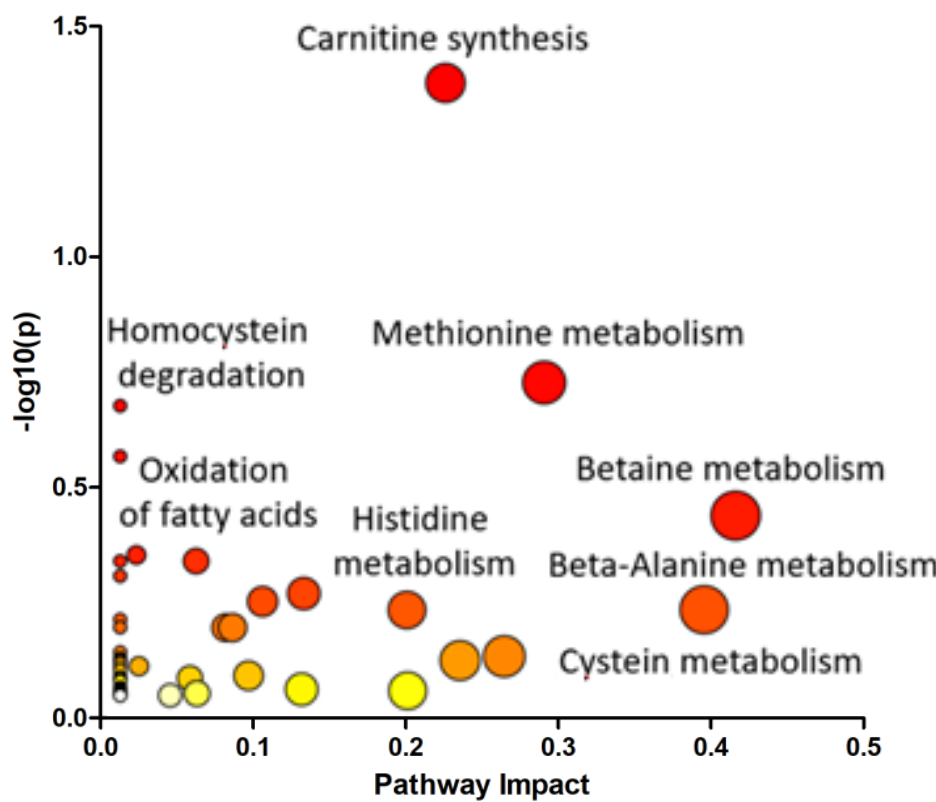

d)

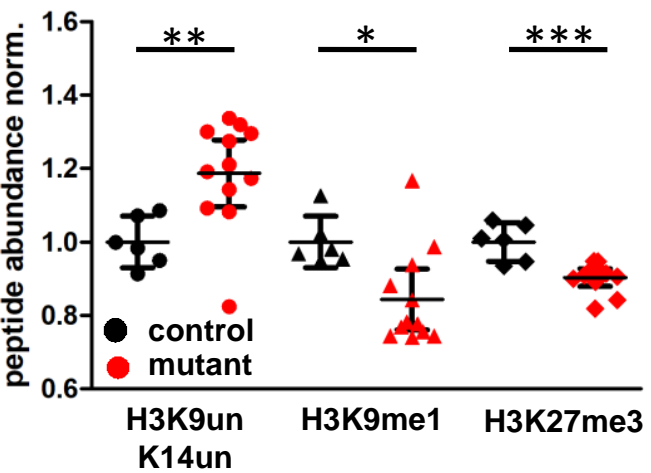

\section{Global Metabolomics}

Pathway Impact

\section{Histone modifications}

K14un

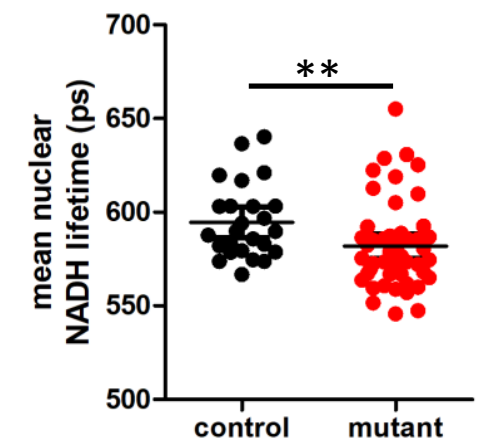


(which was not certified by peer review) is the author/funder, who has granted bioRxiv a license to display the preprint in perpetuity. It is $\mathrm{m}$ available under aCC-BY-NC-ND 4.0 International license.

Fig.3 Calcium homeostasis links paternal HDC variant with the ND5 m.13708G>A-H7 mtDNA
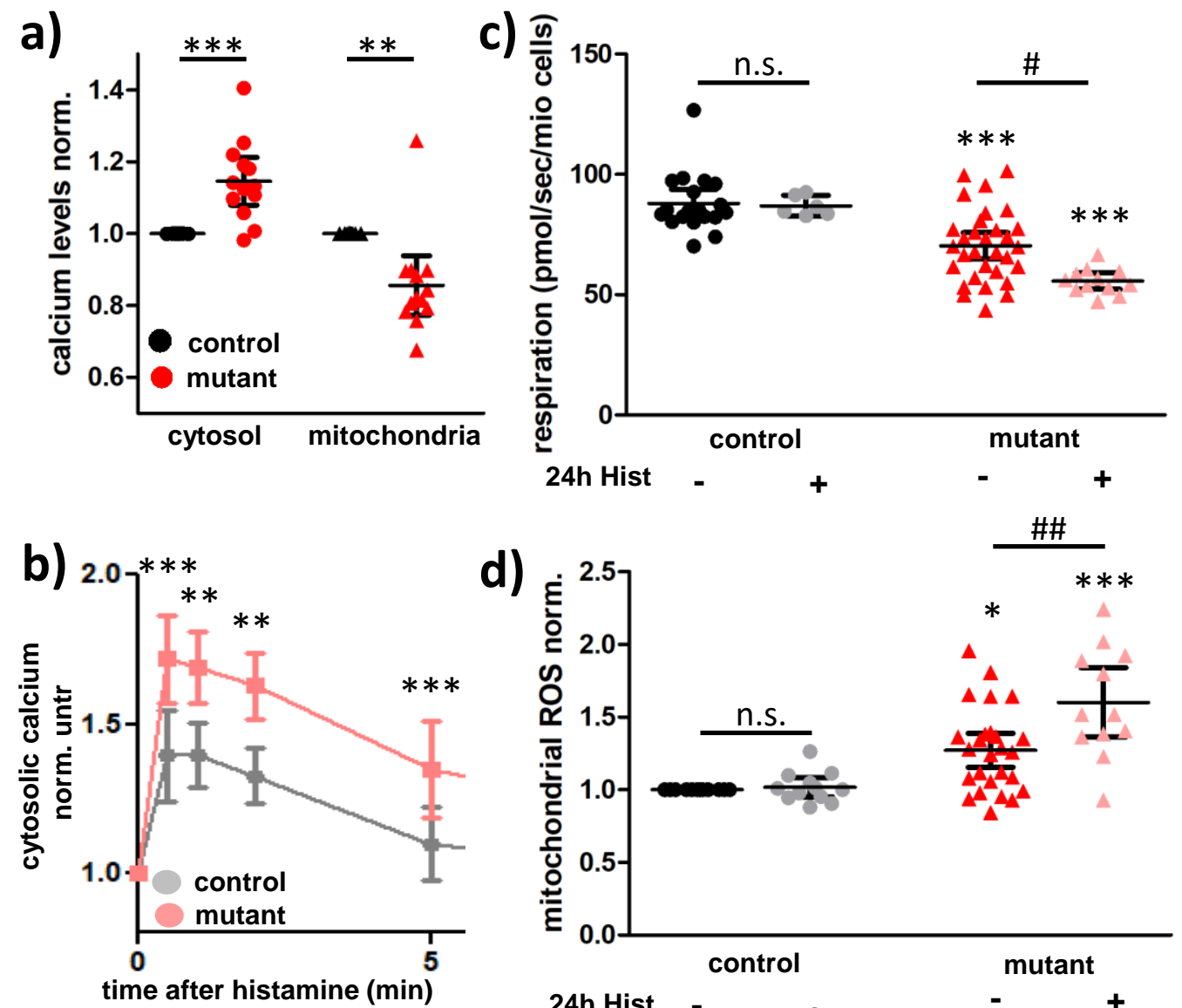

d)

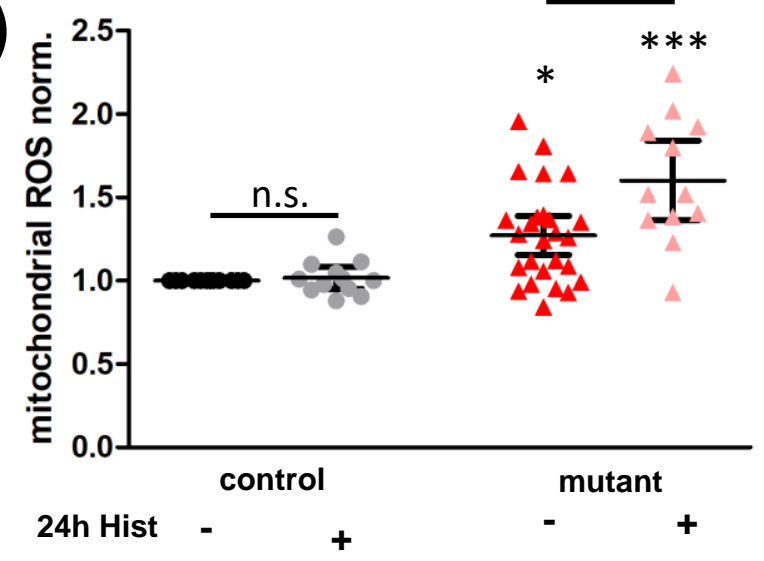

e)

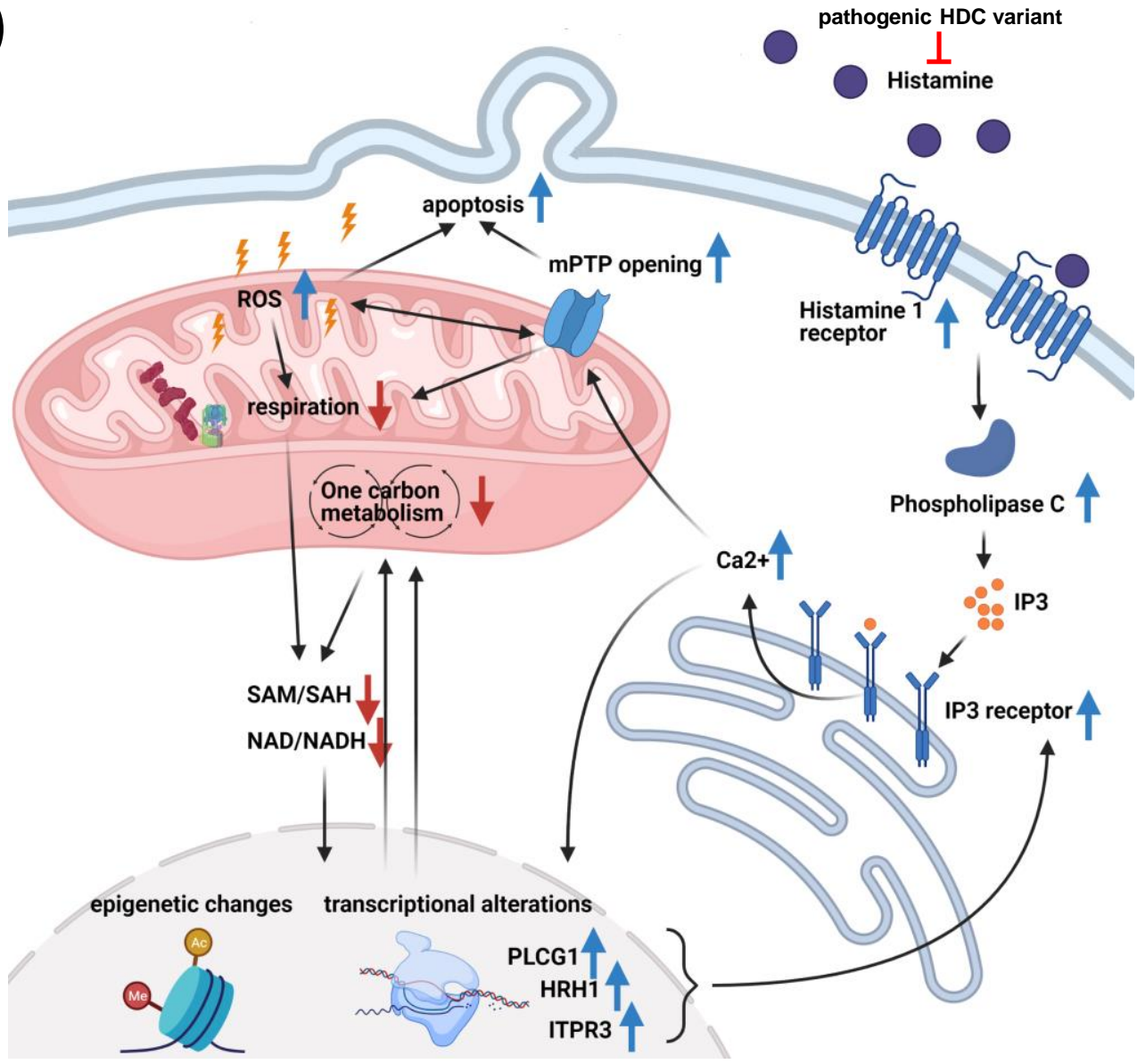


Fig.4 Patient lymphoblasts show increased ROS levels and a switch from complex I to complex II respiration

a)

ETS capacity

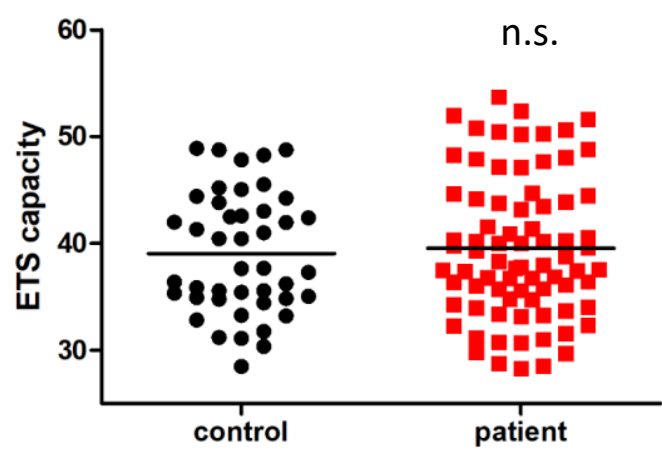

b)

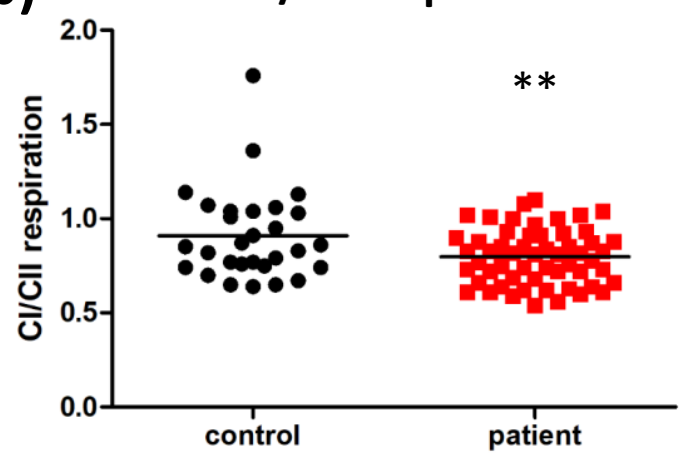

c)

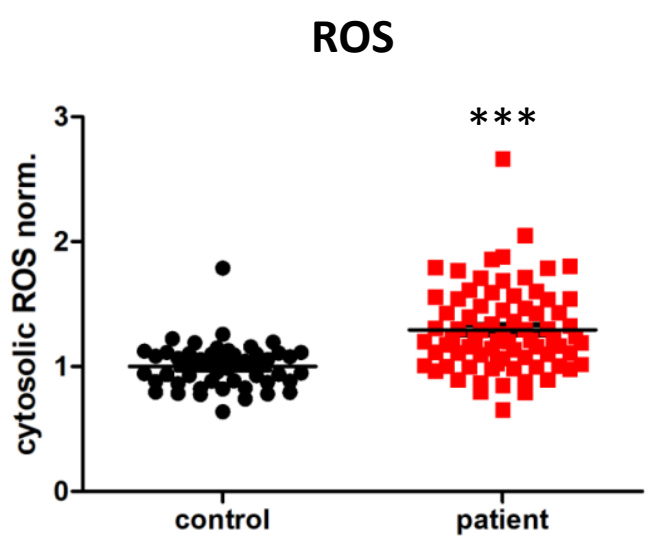

d)
d)
mitochondrial mass

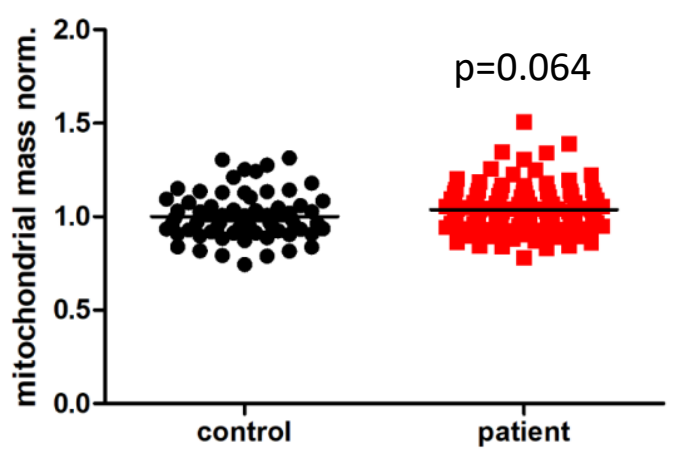

e)

ETS capacity

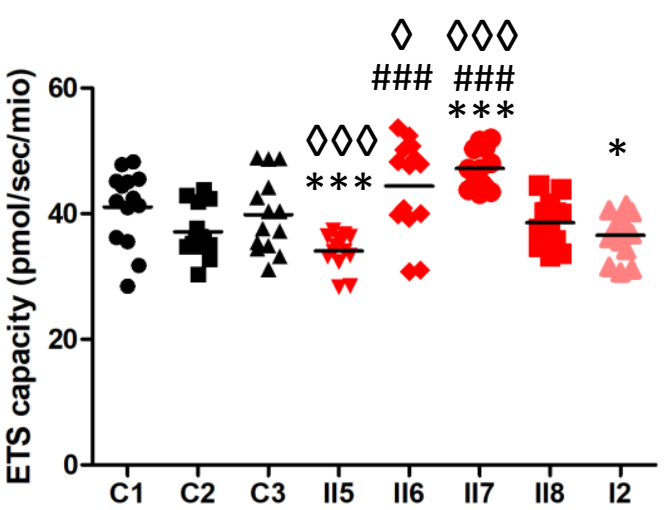

f)

$\mathrm{Cl} / \mathrm{Cll}$ respiration

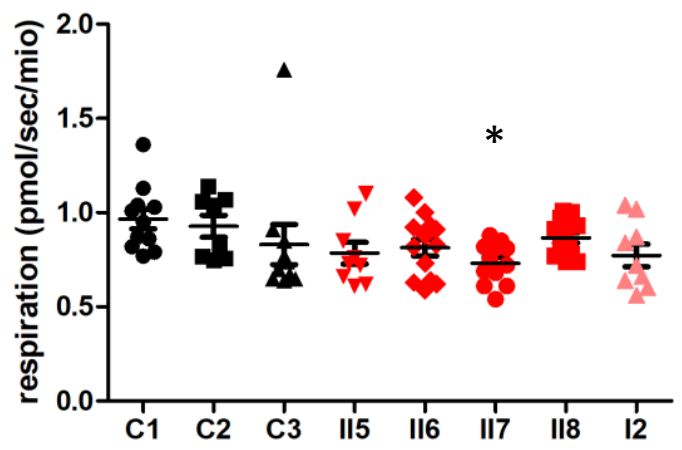

g)

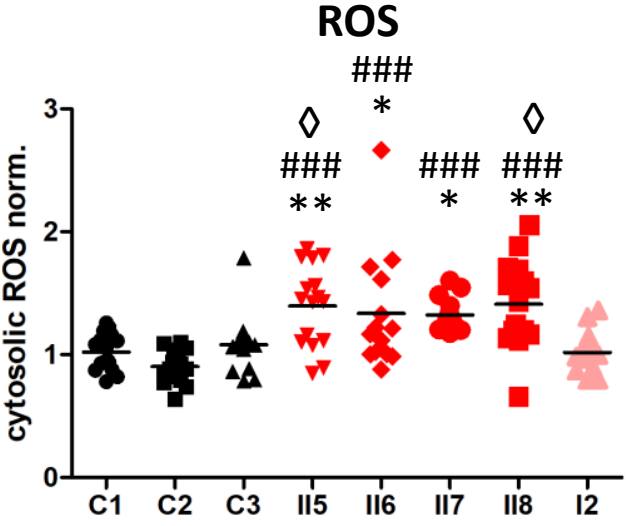

h)

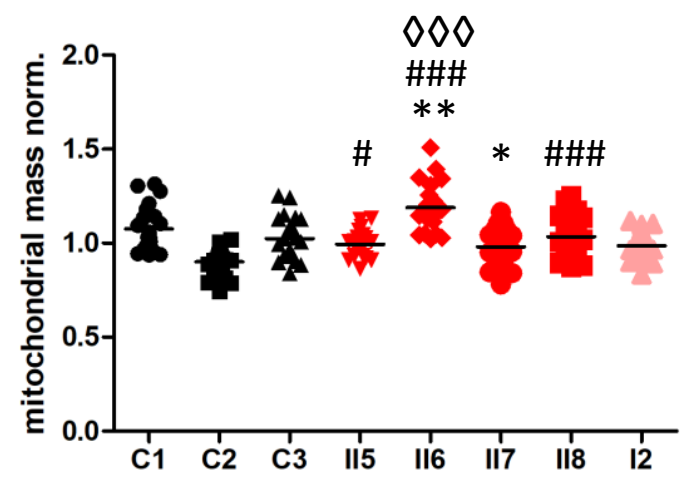




\section{Fig.S1 Mitochondrial haplogroup J defining variant ND5}

\section{m.13708G >A on haplogroup H7 background}

\begin{tabular}{|c|c|c|c|c|c|}
\hline control & patient & locus & nucleotide & Amino acid & overall \\
\hline variants & variants & & change & change & frequency \\
\hline 263 & 263 & D-Loop & $A \rightarrow G$ & N/A & $94.97 \%$ \\
\hline 315 & 315 & D-Loop & Ins C & $\mathrm{N} / \mathrm{A}$ & $30.72 \%$ \\
\hline 750 & 750 & RNR1 & $A \rightarrow G$ & $\mathrm{~N} / \mathrm{A}$ & $98.28 \%$ \\
\hline 1438 & 1438 & RNR1 & $A \rightarrow G$ & N/A & $94.85 \%$ \\
\hline 1719 & & RNR2 & $\mathrm{G} \rightarrow \mathrm{A}$ & $\mathrm{N} / \mathrm{A}$ & $4.86 \%$ \\
\hline 4769 & 4769 & ND2 & $A \rightarrow G$ & no & $97.60 \%$ \\
\hline \multirow[t]{2}{*}{4793} & 4793 & ND2 & $A \rightarrow G$ & no & $0.75 \%$ \\
\hline & 6152 & $\mathrm{CO} 1$ & $T \rightarrow C$ & no & $0.80 \%$ \\
\hline \multirow[t]{3}{*}{8860} & 8860 & & $A \rightarrow G$ & yes & $98.48 \%$ \\
\hline & 11827 & ND4 & $\mathrm{T} \rightarrow \mathrm{C}$ & no & $0.16 \%$ \\
\hline & 13708 & ND5 & $G \rightarrow A$ & yes & $7.18 \%$ \\
\hline \multirow[t]{3}{*}{15326} & 15326 & & $A \rightarrow G$ & yes & $98.66 \%$ \\
\hline & 15409 & CYB & $C \rightarrow T$ & no & $0.07 \%$ \\
\hline & 16193 & D-Loop & $C \rightarrow T$ & $\mathrm{~N} / \mathrm{A}$ & $1.30 \%$ \\
\hline 16256 & & D-Loop & $C \rightarrow T$ & $\mathrm{~N} / \mathrm{A}$ & $3.23 \%$ \\
\hline 16261 & & D-Loop & $C \rightarrow T$ & N/A & $7.48 \%$ \\
\hline \multirow[t]{2}{*}{16294} & & D-Loop & $C \rightarrow T$ & $\mathrm{~N} / \mathrm{A}$ & $8.92 \%$ \\
\hline & 16311 & D-Loop & $\mathrm{T} \rightarrow \mathrm{C}$ & N/A & $19.81 \%$ \\
\hline 16519 & 16519 & D-loop & $T \rightarrow C$ & N/A & $62.94 \%$ \\
\hline
\end{tabular}

b)

\section{c)}

\begin{tabular}{|c|c|c|c|c|c|c|c|c|c|c|c|c|}
\hline \multicolumn{13}{|c|}{ co-occurrence frequency normalized } \\
\hline alleles & counts & 13708A & $8860 G$ & $15326 G$ & 73A & 2706A & $4216 \mathrm{~T}$ & $7028 \mathrm{C}$ & 10398A & $11719 G$ & $14766 \mathrm{C}$ & $15452 C$ \\
\hline 13708A & 3722 & & 1.01 & 1.01 & 0.31 & 0.29 & 0.37 & 0.27 & 0.40 & 0.26 & 0.23 & 0.37 \\
\hline $8860 G$ & 51048 & 1.01 & & 1.01 & 0.98 & 0.97 & 1.00 & 0.96 & 0.99 & 0.97 & 0.97 & 1.00 \\
\hline $15326 G$ & 51143 & 1.01 & 1.01 & & 0.98 & 0.97 & 1.00 & 0.97 & 0.99 & 0.97 & 0.97 & 1.00 \\
\hline 73A & 12410 & 0.31 & 0.98 & 0.98 & & 3.50 & 1.09 & 3.81 & 1.62 & 3.84 & 3.69 & 1.09 \\
\hline 2706A & 10904 & 0.29 & 0.96 & 0.97 & 3.50 & & 1.10 & 4.69 & 1.64 & 3.97 & 3.91 & 1.10 \\
\hline $4216 \mathrm{~T}$ & 46679 & 0.37 & 1.00 & 1.00 & 1.09 & 1.10 & & 1.10 & 1.01 & 1.10 & 1.10 & 1.10 \\
\hline 7028C & 9977 & 0.27 & 0.96 & 0.97 & 3.81 & 4.69 & 1.10 & & 1.78 & 4.33 & 4.27 & 1.10 \\
\hline 10398A & 29035 & 0.40 & 0.99 & 0.99 & 1.62 & 1.64 & 1.01 & 1.78 & & 1.78 & 1.72 & 1.01 \\
\hline $11719 G$ & 11691 & 0.26 & 0.97 & 0.97 & 3.84 & 3.97 & 1.10 & 4.33 & 1.78 & & 4.19 & 1.10 \\
\hline $14766 C$ & 11977 & 0.23 & 0.97 & 0.97 & 3.69 & 3.91 & 1.10 & 4.27 & 1.72 & 4.19 & & 1.10 \\
\hline $15452 C$ & 46988 & 0.37 & 1.00 & 1.00 & 1.09 & 1.10 & 1.10 & 1.10 & 1.01 & 1.10 & 1.10 & \\
\hline
\end{tabular}




\section{Fig.S2 ND5 m.13708G>A-H7 mtDNA reduces bioenergetic function}

a)

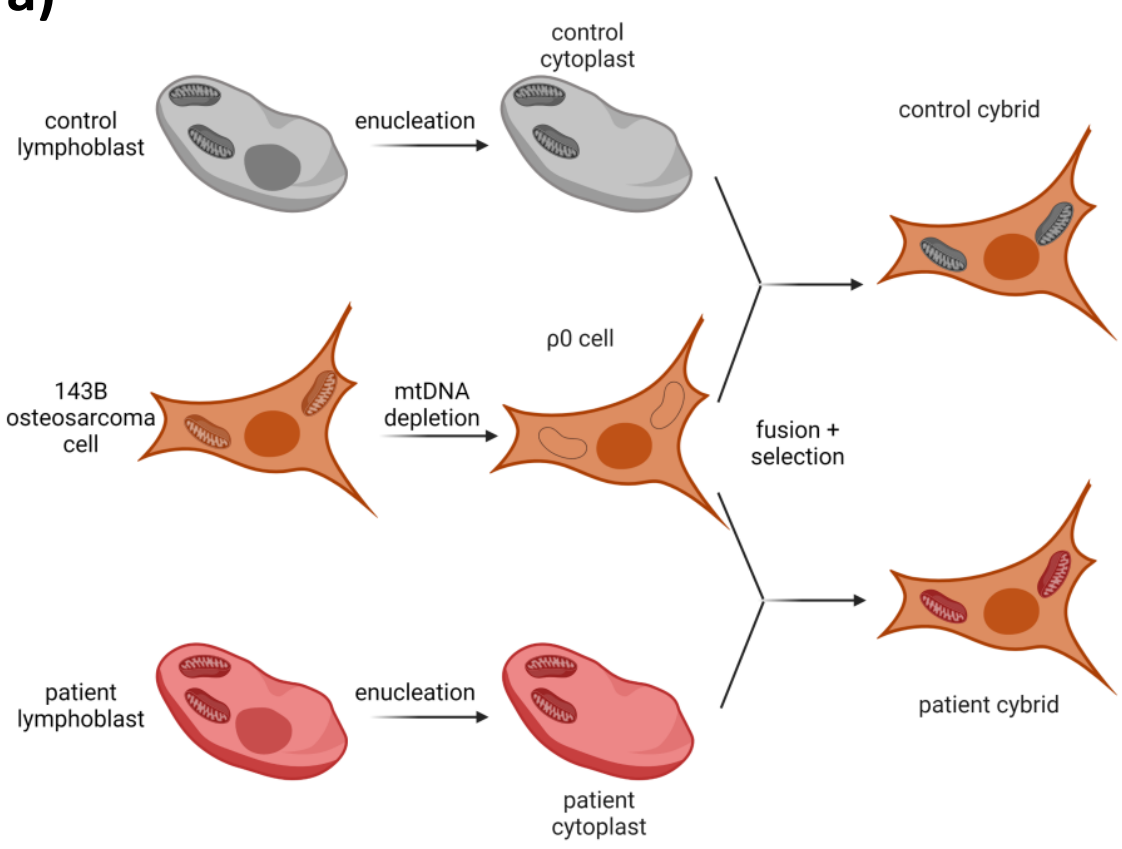

d

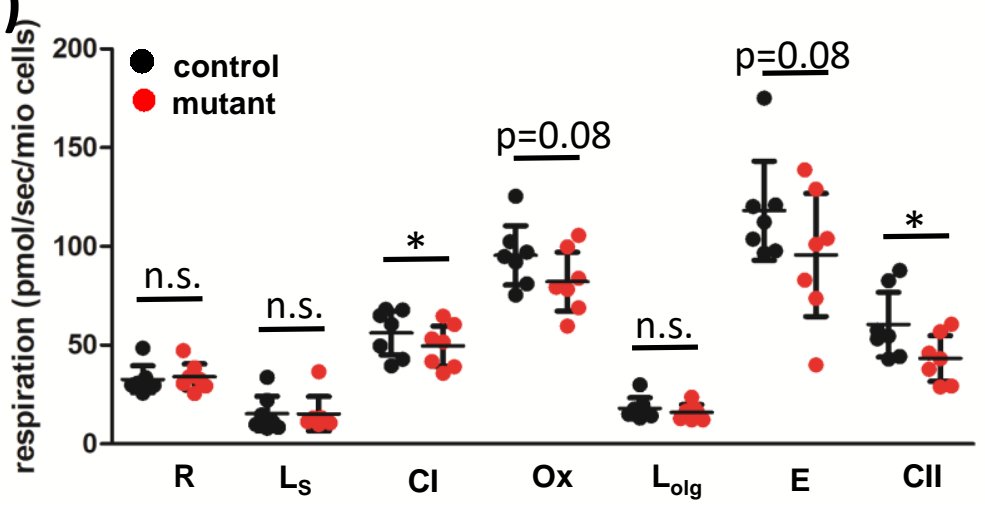

f)

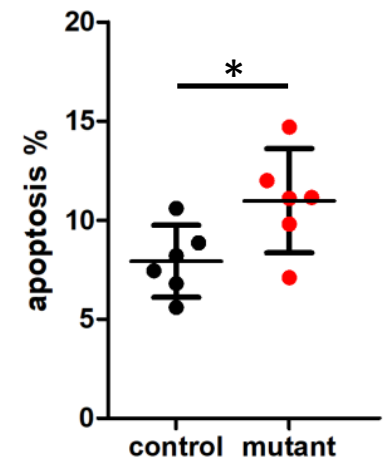

b)

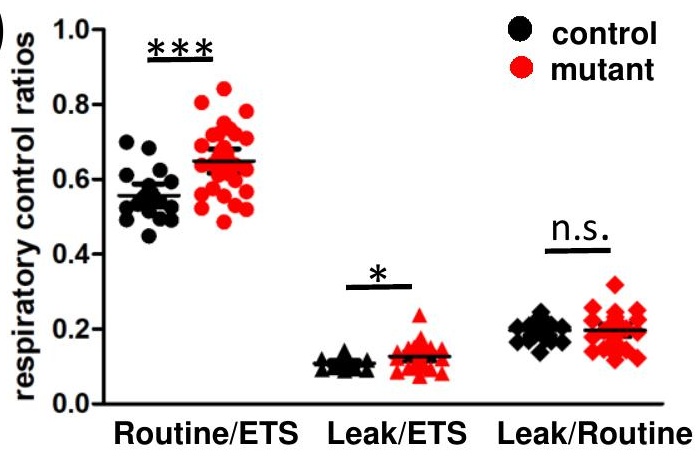

c)

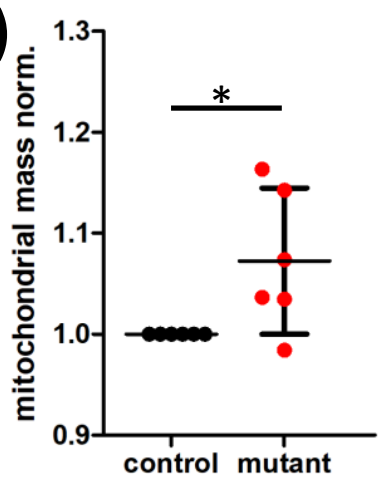

e)

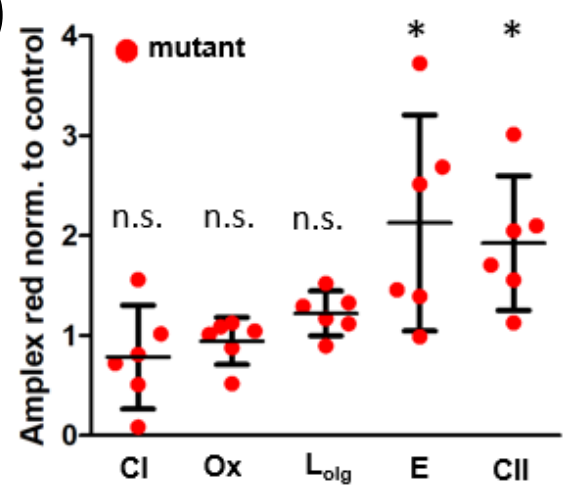


Fig.S3 Mitochondrial haplogroup J shows similar bioenergetics to haplogroup H

a)

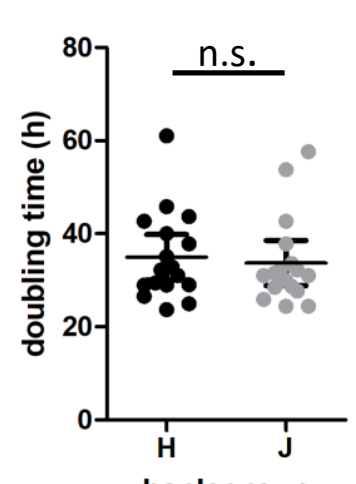

haplogroup

d)

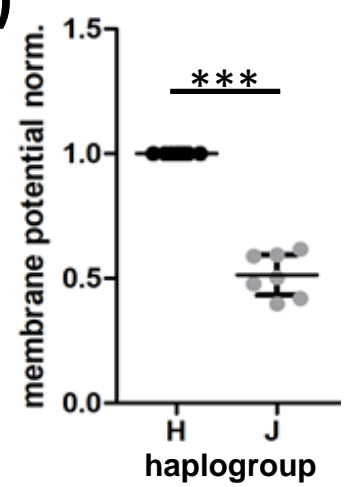

b)

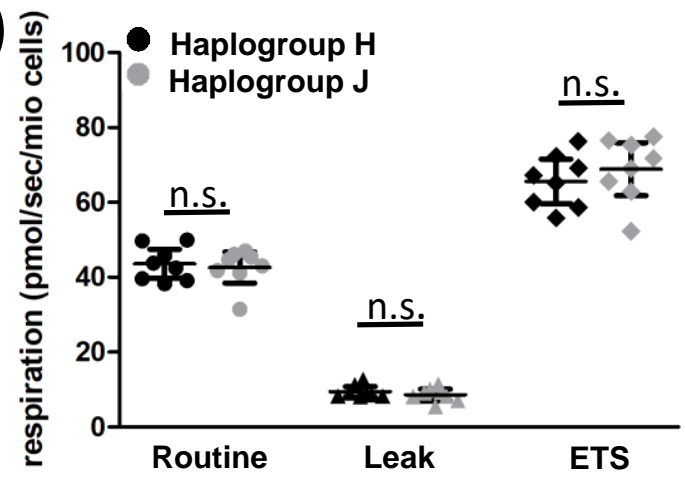

e)

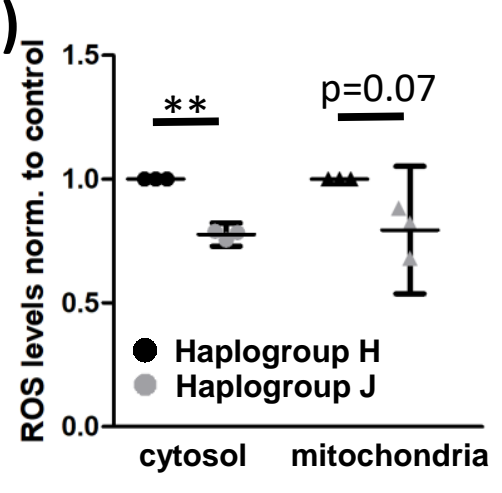

c)

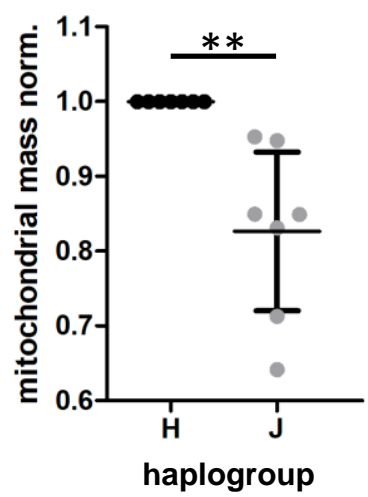

f)

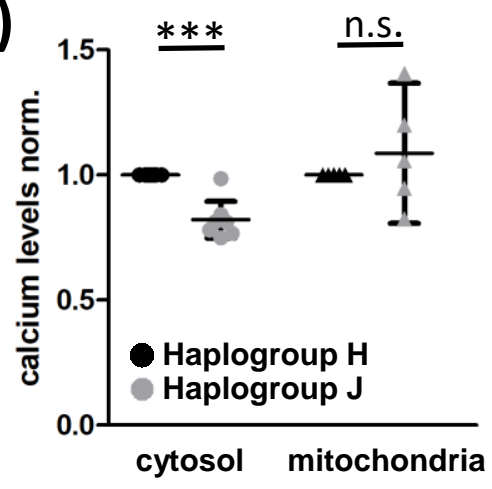


Fig.S4 ND5 m.13708G>A-H7 mtDNA alters nicotinamide, TCA cycle and one carbon metabolism

a)

\begin{tabular}{|c|c|c|}
\hline gene & $\log F C$ & adj p-value \\
\hline NADK & 0.23 & $1.90 \mathrm{E}-04$ \\
\hline NMNAT1 & 0.27 & 2.07E-03 \\
\hline NAMPT & 0.09 & 2.19E-02 \\
\hline NT5M & 0.24 & 4.59E-02 \\
\hline ENPP1 & 0.43 & $7.28 \mathrm{E}-02$ \\
\hline NT5C & 0.08 & $1.92 \mathrm{E}-01$ \\
\hline NUDT12 & 0.05 & 5.56E-01 \\
\hline NMRK1 & 0.05 & $6.32 \mathrm{E}-01$ \\
\hline NT5C3A & 0.00 & 9.59E-01 \\
\hline NMNAT2 & -0.02 & 8.94E-01 \\
\hline NADSYN & & \\
\hline 1 & -0.10 & 9.11E-02 \\
\hline PNP & -0.09 & 3.15E-02 \\
\hline NNT & -0.32 & $3.36 \mathrm{E}-03$ \\
\hline BST1 & -0.79 & 5.24E-04 \\
\hline NNMT & -2.25 & 3.19E-05 \\
\hline NT5E & -0.49 & 7.00E-07 \\
\hline AOX1 & -1.61 & 2.41E-08 \\
\hline NT5C2 & -0.50 & 1.75E-09 \\
\hline
\end{tabular}

b)

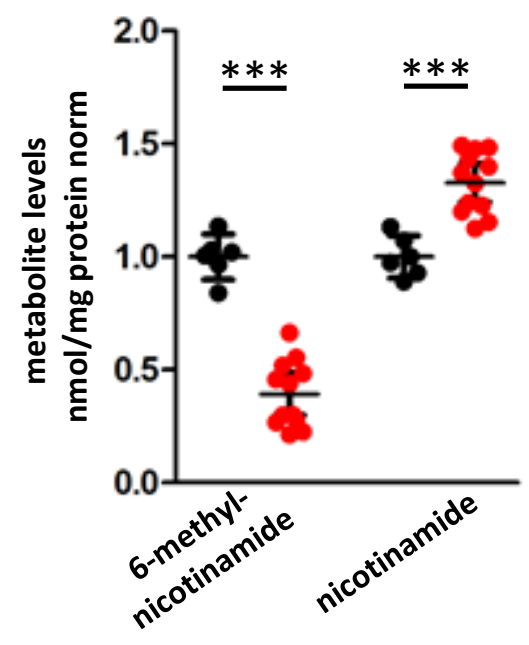

d)

\section{KEGG One Carbon \\ Pool by Folate}

\begin{tabular}{|c|c|c|}
\hline gene & logFC & adj p-value \\
\hline TYMS & 0.31 & $1.54 \mathrm{E}-03$ \\
\hline MTHFS & 0.20 & $3.73 \mathrm{E}-03$ \\
\hline MTR & 0.18 & $5.75 \mathrm{E}-04$ \\
\hline MTHFD1 & 0.09 & $1.70 \mathrm{E}-01$ \\
\hline MTHFD2L & 0.02 & $8.63 \mathrm{E}-01$ \\
\hline ATIC & -0.01 & $8.16 \mathrm{E}-01$ \\
\hline SHMT1 & -0.03 & $5.68 \mathrm{E}-01$ \\
\hline MTFMT & -0.08 & $3.76 \mathrm{E}-01$ \\
\hline MTHFR & -0.13 & $1.52 \mathrm{E}-01$ \\
\hline ALDH1L1 & -1.93 & $5.15 \mathrm{E}-02$ \\
\hline GART & -0.06 & $1.72 \mathrm{E}-02$ \\
\hline DHFR & -0.41 & $5.18 \mathrm{E}-03$ \\
\hline MTHFD1L & -0.22 & $3.13 \mathrm{E}-05$ \\
\hline CBS & -0.16 & $5.13 \mathrm{E}-05$ \\
\hline SHMT2 & -0.39 & $2.48 \mathrm{E}-06$ \\
\hline MTHFD2 & -0.47 & $1.21 \mathrm{E}-06$ \\
\hline
\end{tabular}

e) Targeted metabolomics 1C metabolism

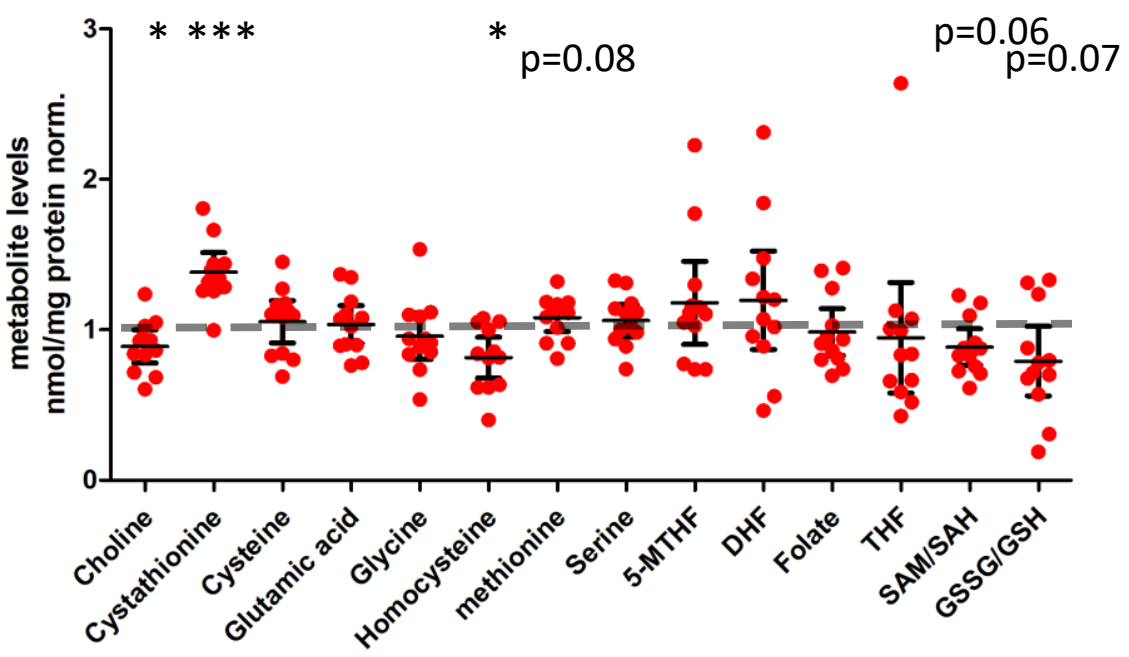

c)

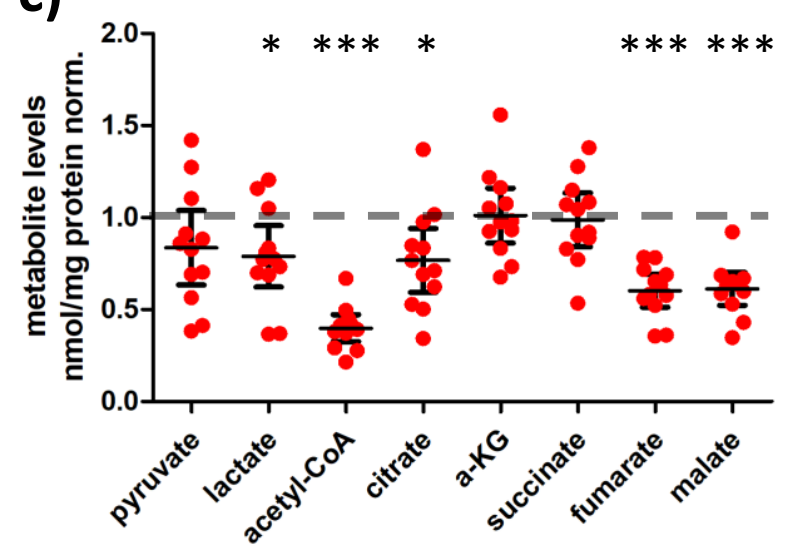


Fig.S5 ND5 m.13708G>A-H7 mtDNA results in histone hypomethylation

a) Upregulated modifications

\begin{tabular}{|c|c|c|c|}
\hline modification & fold change & ratio & p-value \\
\hline H3K4un & 1.02 & 0.838 & 0.0003 \\
\hline H3.3К27unK36un & 1.35 & 0.120 & 0.0006 \\
\hline H4K16ас & 1.08 & 0.287 & 0.0058 \\
\hline H3K9me2 & 1.11 & 0.122 & 0.0083 \\
\hline H3K9unK14un & 1.19 & 0.218 & 0.0087 \\
\hline H3К36me1 & 1.15 & 0.128 & 0.0177 \\
\hline
\end{tabular}

\section{b)}

Downregulated modifications

\begin{tabular}{|c|c|c|c|}
\hline modification & fold change & ratio & p-value \\
\hline H3K27me3 & 0.90 & 0.105 & 0.0003 \\
\hline H3K4me1 & 0.90 & 0.160 & 0.0003 \\
\hline H3K18acK23ac & 0.78 & 0.041 & 0.0013 \\
\hline H3K18ac & 0.80 & 0.043 & 0.0023 \\
\hline H3K9me3K14ac & 0.91 & 0.016 & 0.0029 \\
\hline H3K4me3 & 0.83 & 0.001 & 0.0036 \\
\hline H3K4me2 & 0.84 & 0.001 & 0.0043 \\
\hline H3.3K27me3 & 0.70 & 0.201 & 0.0136 \\
\hline H3K9me1 & 0.84 & 0.321 & 0.0149 \\
\hline H3K9me1K14ac & 0.89 & 0.141 & 0.0160 \\
\hline H3K27me3K36me & & & \\
\hline 1 & 0.84 & 0.012 & 0.0308 \\
\hline H4K20me1 & 0.76 & 0.332 & 0.0384 \\
\hline
\end{tabular}

\section{unmodified peptide}

methylation

acetylation

methylation+acetylation c) Single PTMs

\begin{tabular}{|c|c|c|c|}
\hline modification & \multicolumn{1}{c}{ fold change } & \multicolumn{1}{c|}{ ratio } & p-value \\
\hline H4K16ac & 1.07 & 0.444 & 0.0253 \\
\hline H3K9me2 & 1.07 & 0.161 & 0.0368 \\
\hline H3K36me1 & 1.05 & 0.261 & 0.1134 \\
\hline H4K20me2 & 1.23 & 0.383 & 0.1947 \\
\hline H4K20me3 & 1.35 & 0.005 & 0.1957 \\
\hline H3K9me3 & 1.04 & 0.083 & 0.2399 \\
\hline H3K9ac & 1.47 & 0.004 & 0.3102 \\
\hline H3K4ac & 2.05 & 0.000 & 0.5182 \\
\hline H3K27me1 & 1.01 & 0.107 & 0.8335 \\
\hline H3K23me1 & 0.99 & 0.001 & 0.9592 \\
\hline H3K27me2 & 0.99 & 0.419 & 0.8015 \\
\hline H3K18me1 & 0.93 & 0.001 & 0.7446 \\
\hline H4K12ac & 0.98 & 0.185 & 0.7172 \\
\hline H3K36me3 & 0.94 & 0.057 & 0.6864 \\
\hline H4K5ac & 0.96 & 0.067 & 0.6543 \\
\hline H3K36me2 & 0.87 & 0.027 & 0.5787 \\
\hline H3K23ac & 0.96 & 0.339 & 0.4839 \\
\hline H4K8ac & 0.96 & 0.111 & 0.4813 \\
\hline H3K14ac & 0.96 & 0.271 & 0.1781 \\
\hline H3K27ac & 0.84 & 0.003 & 0.1333 \\
\hline H4K20me1 & 0.76 & 0.332 & 0.0384 \\
\hline H3K9me1 & 0.86 & 0.462 & 0.0085 \\
\hline H3K4me2 & 0.84 & 0.001 & 0.0043 \\
\hline H3K4me3 & 0.83 & 0.001 & 0.0036 \\
\hline H3K18ac & 0.79 & 0.084 & 0.0004 \\
\hline H3K4me1 & 0.90 & 0.160 & 0.0003 \\
\hline H3K27me3 & 0.90 & 0.117 & 0.0003 \\
\hline & & & \\
\hline & & & \\
\hline
\end{tabular}


Fig.S6 ND5 m.13708G>A-H7 mtDNA alters cholesterol, ketone and collagen metabolism

a) Reactome Cholesterol Biosynthesis

\begin{tabular}{|c|c|c|}
\hline \multirow{3}{*}{$\begin{array}{l}\text { gene } \\
\text { HSD17B }\end{array}$} & $\log F C$ & adj $p$-value \\
\hline & & \\
\hline & 0.63 & $6.91 E-11$ \\
\hline ACAT2 & 0.59 & $4.55 E-10$ \\
\hline DHCR7 & 0.64 & 8.25E-10 \\
\hline SQLE & 0.48 & 2.16E-08 \\
\hline LSS & 0.52 & 2.41E-08 \\
\hline NSDHL & 0.40 & 2.57E-08 \\
\hline MSM01 & 0.49 & $3.16 \mathrm{E}-08$ \\
\hline FDFT1 & 0.56 & 4.39E-08 \\
\hline HMGCS1 & 0.72 & 8.91E-08 \\
\hline MVD & 0.49 & $1.98 \mathrm{E}-06$ \\
\hline MVK & 0.48 & 2.81E-06 \\
\hline DHCR24 & 0.34 & 2.35E-05 \\
\hline CYP51A & & \\
\hline 1 & 0.29 & 2.90E-04 \\
\hline FDPS & 0.20 & 3.85E-04 \\
\hline \begin{tabular}{|l|} 
TM7SF2 \\
\end{tabular} & 0.39 & 7.49E-03 \\
\hline SC5D & 0.13 & 2.07E-02 \\
\hline \begin{tabular}{|l|} 
HMGCR \\
\end{tabular} & 0.27 & 2.69E-02 \\
\hline IDI1 & 0.06 & 1.61E-01 \\
\hline LBR & 0.00 & $9.80 \mathrm{E}-01$ \\
\hline IDI2 & & \\
\hline PLPP6 & & \\
\hline PMVK & -0.01 & 9.02E-01 \\
\hline EBP & -0.03 & 7.32E-01 \\
\hline GGPS1 & -0.09 & 1.74E-01 \\
\hline ARV1 & -0.15 & $1.20 \mathrm{E}-02$ \\
\hline
\end{tabular}

d)

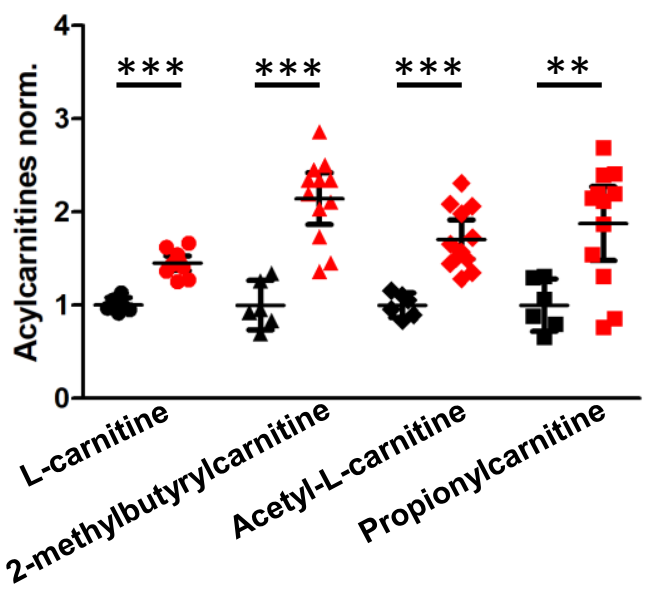

b) Reactome Ketone Body Metabolism

\begin{tabular}{|c|c|c|}
\hline gene & logFC & adj $p$-value \\
\hline AACS & 0.35 & $1.27 \mathrm{E}-05$ \\
\hline HMGCL & 0.17 & $5.42 \mathrm{E}-03$ \\
\hline BDH1 & 0.14 & $1.49 \mathrm{E}-02$ \\
\hline $\begin{array}{c}\text { HMGCLL } \\
1\end{array}$ & & \\
\hline HMGCS2 & & \\
\hline OXCT2 & & \\
\hline ACAT1 & -0.14 & $2.69 \mathrm{E}-03$ \\
\hline BDH2 & -0.31 & $1.69 \mathrm{E}-03$ \\
\hline OXCT1 & -0.26 & $7.61 \mathrm{E}-04$ \\
\hline ACSS3 & -0.91 & $1.35 \mathrm{E}-07$ \\
\hline
\end{tabular}

c) Reactome Collagen Formation

\begin{tabular}{|c|c|c|}
\hline gene & logFC & adj p-value \\
\hline SERPINH1 & 0.33 & $2.61 \mathrm{E}-06$ \\
\hline LOXL2 & 0.48 & $8.67 \mathrm{E}-06$ \\
\hline PLOD3 & 0.23 & $1.28 \mathrm{E}-04$ \\
\hline MMP13 & 0.75 & $2.23 \mathrm{E}-04$ \\
\hline P3H1 & 0.16 & $4.40 \mathrm{E}-04$ \\
\hline LOX & 0.42 & $2.47 \mathrm{E}-03$ \\
\hline P4HB & 0.08 & $2.35 \mathrm{E}-02$ \\
\hline PLOD2 & 0.14 & $2.59 \mathrm{E}-02$ \\
\hline COL27A1 & 0.33 & $2.74 \mathrm{E}-02$ \\
\hline PLEC & 0.13 & $3.57 \mathrm{E}-02$ \\
\hline COL5A1 & 0.36 & $3.88 \mathrm{E}-02$ \\
\hline LAMA3 & -0.32 & $3.01 \mathrm{E}-02$ \\
\hline CTSL & -0.09 & $2.83 \mathrm{E}-02$ \\
\hline COL9A3 & -0.18 & $2.64 \mathrm{E}-02$ \\
\hline LAMC2 & -0.34 & $2.49 \mathrm{E}-02$ \\
\hline COL4A6 & -0.97 & $1.60 \mathrm{E}-02$ \\
\hline PXDN & -0.14 & $9.39 \mathrm{E}-03$ \\
\hline LOXL1 & -0.45 & $8.99 \mathrm{E}-03$ \\
\hline COLGALT2 & -0.44 & $3.81 \mathrm{E}-03$ \\
\hline COL6A2 & -0.36 & $2.47 \mathrm{E}-03$ \\
\hline COL24A1 & -0.71 & $2.09 \mathrm{E}-03$ \\
\hline ADAMTS3 & -0.32 & $1.20 \mathrm{E}-03$ \\
\hline ITGB4 & -0.81 & $6.50 \mathrm{E}-04$ \\
\hline COL4A5 & -0.44 & $4.78 \mathrm{E}-04$ \\
\hline COL12A1 & -0.64 & $4.31 \mathrm{E}-04$ \\
\hline PCOLCE & -0.53 & $5.05 \mathrm{E}-05$ \\
\hline LAMB3 & -0.88 & $5.00 \mathrm{E}-05$ \\
\hline ITGA6 & -0.72 & $2.61 \mathrm{E}-05$ \\
\hline COL8A1 & -0.97 & $1.00 \mathrm{E}-05$ \\
\hline CTSB & -0.39 & $3.87 \mathrm{E}-06$ \\
\hline COL13A1 & -0.72 & $5.71 \mathrm{E}-10$ \\
\hline & & \\
\hline
\end{tabular}


Fig.S7 ND5 m.13708G>A-H7 mtDNA reduces mitochondrial calcium uptake and increases histamine sensitivity

a) Reactome Mitochondrial calcium ion transport

\begin{tabular}{|c|c|c|}
\hline \multicolumn{1}{|c|}{ gene } & \multicolumn{1}{c}{ logFC } & adj p-value \\
\hline LETM1 & 0.20 & $8.90 \mathrm{E}-05$ \\
\hline STOML2 & 0.19 & $4.06 \mathrm{E}-04$ \\
\hline PMPCA & 0.18 & $7.64 \mathrm{E}-03$ \\
\hline PHB & 0.15 & $2.23 \mathrm{E}-02$ \\
\hline AFG3L2 & 0.12 & $2.62 \mathrm{E}-02$ \\
\hline MICU2 & 0.07 & $7.27 \mathrm{E}-02$ \\
\hline PARL & 0.06 & $2.10 \mathrm{E}-01$ \\
\hline PHB2 & 0.05 & $2.35 \mathrm{E}-01$ \\
\hline PMPCB & 0.04 & $3.63 \mathrm{E}-01$ \\
\hline SLC8A3 & & \\
\hline MAIP1 & & \\
\hline MCUB & & \\
\hline MICU3 & -0.10 & $1.85 \mathrm{E}-01$ \\
\hline SLC8B1 & -0.16 & $9.23 \mathrm{E}-02$ \\
\hline AKAP1 & -0.07 & $8.16 \mathrm{E}-02$ \\
\hline SMDT1 & -0.17 & $2.53 \mathrm{E}-02$ \\
\hline SPG7 & -0.28 & $6.48 \mathrm{E}-04$ \\
\hline VDAC3 & -0.55 & $6.22 \mathrm{E}-04$ \\
\hline VDAC2 & -0.28 & $1.58 \mathrm{E}-05$ \\
\hline VDAC1 & -0.41 & $1.53 \mathrm{E}-05$ \\
\hline MCU & -0.35 & $5.44 \mathrm{E}-06$ \\
\hline MICU1 & -0.43 & $4.24 \mathrm{E}-07$ \\
\hline YME1L1 & -0.33 & $6.83 \mathrm{E}-09$ \\
\hline
\end{tabular}
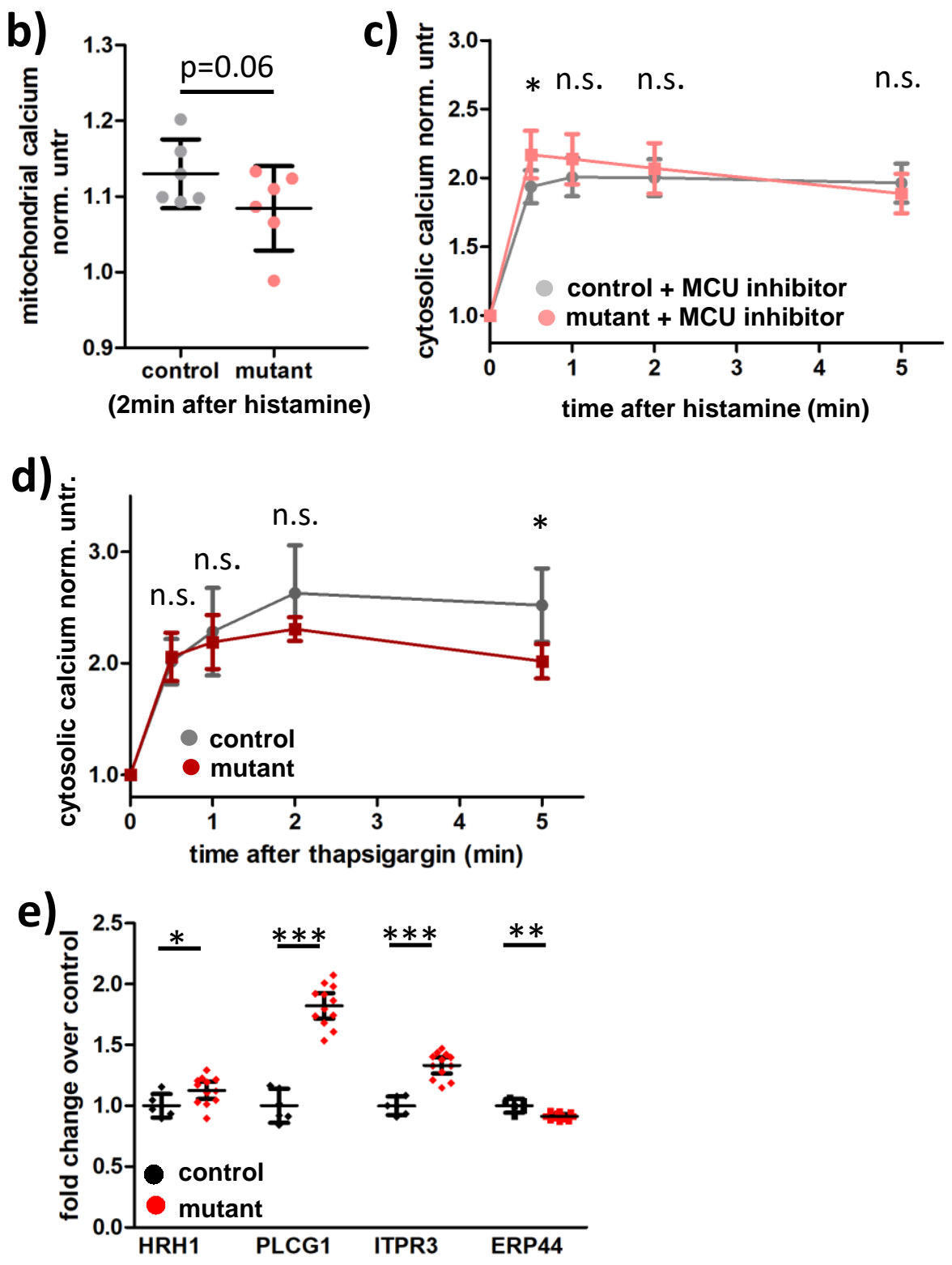
Fig.S8 Histamine toxicity in ND5 m.13708G >A-H7 mtDNA cybrids is mediated via Histamine 1 receptor-mediated calcium release and $\mathrm{mPTP}$ opening

a)

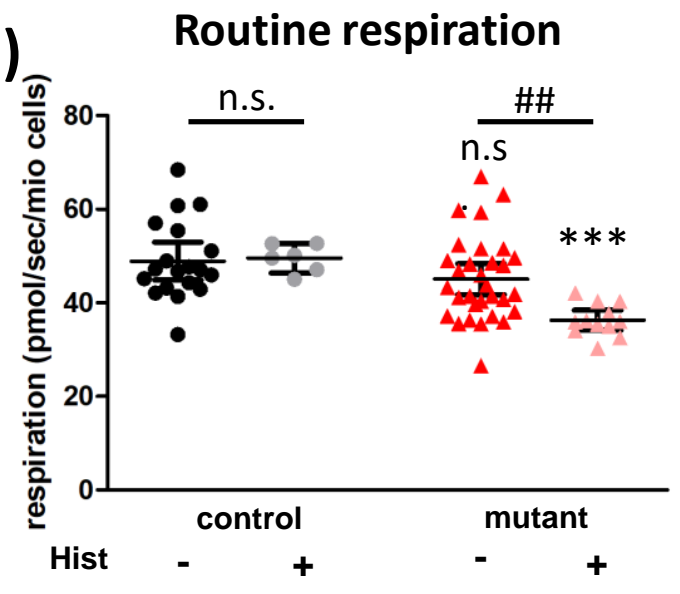

c)

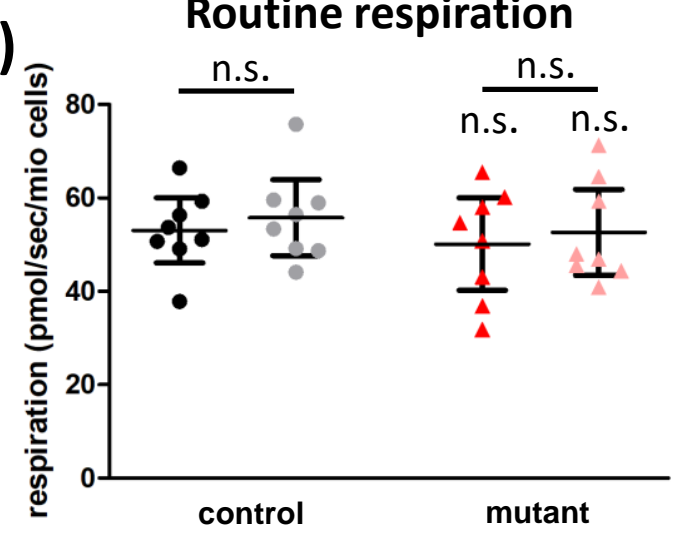

Pyrilamine Hist

Routine respiration

e)

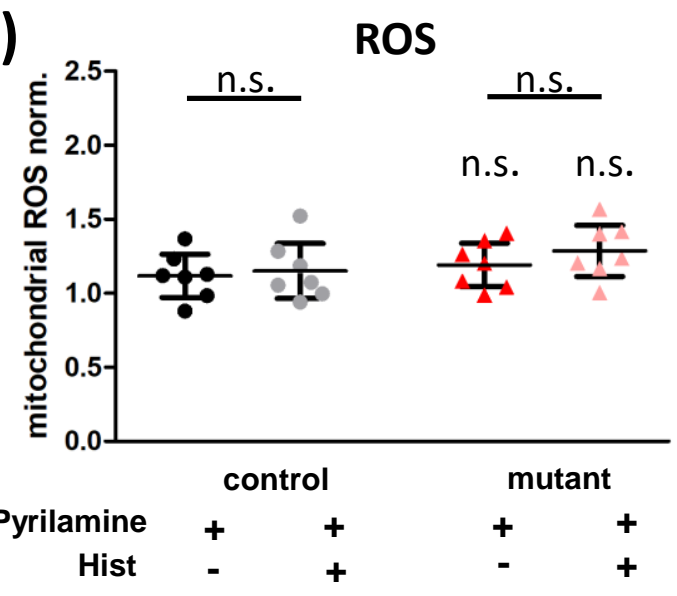

b)

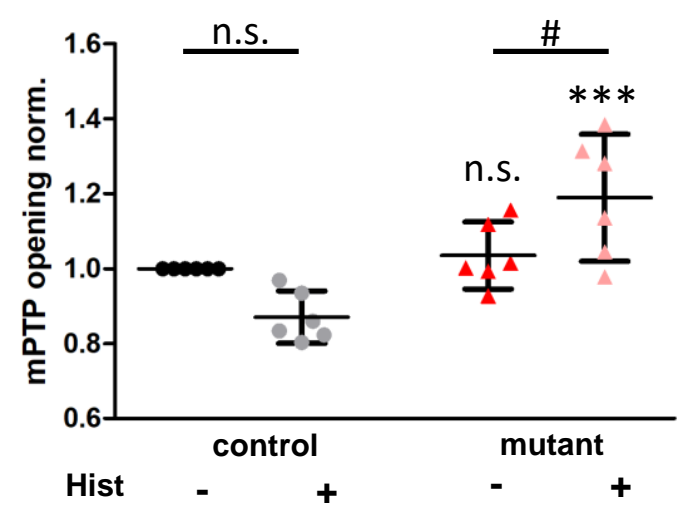

d)

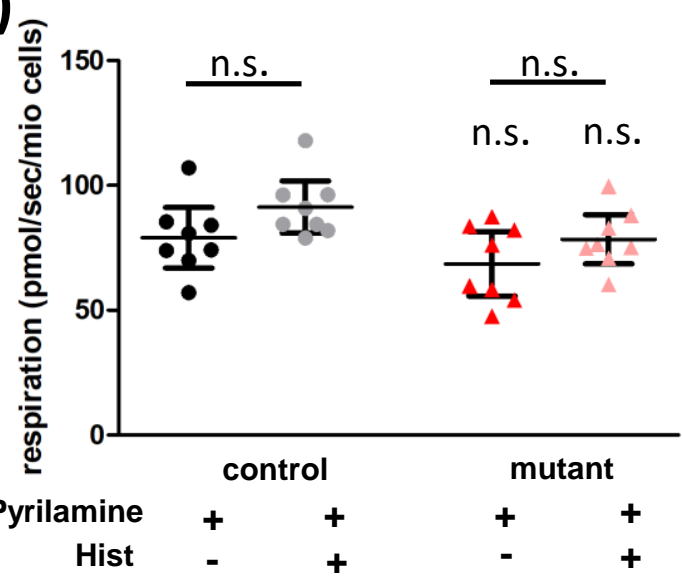


Fig.S9 Histamine 1 receptor inhibition reduced mitochondrial function but alleviates the negative effect of the ND5 m.13708G >A-H7 mtDNA

a)

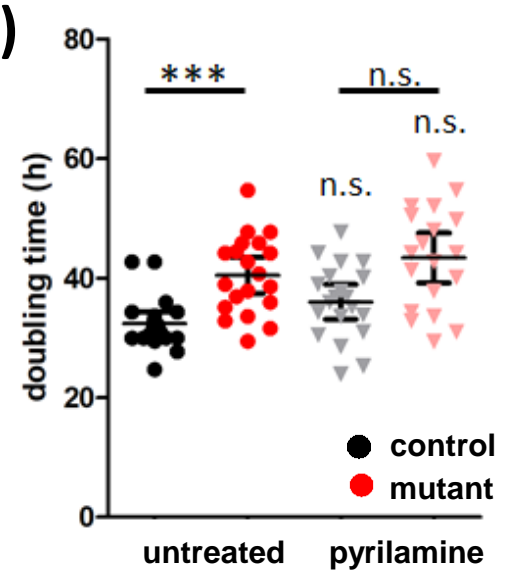

d)

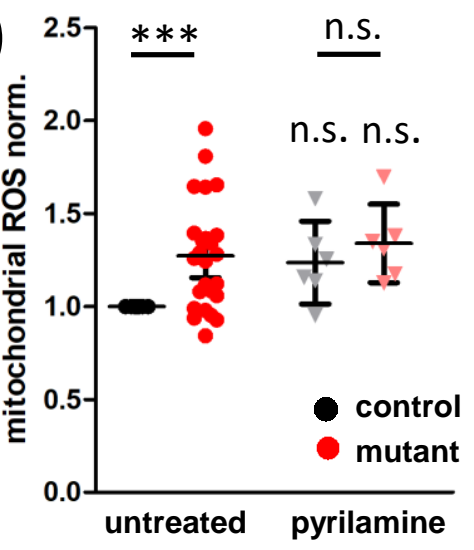

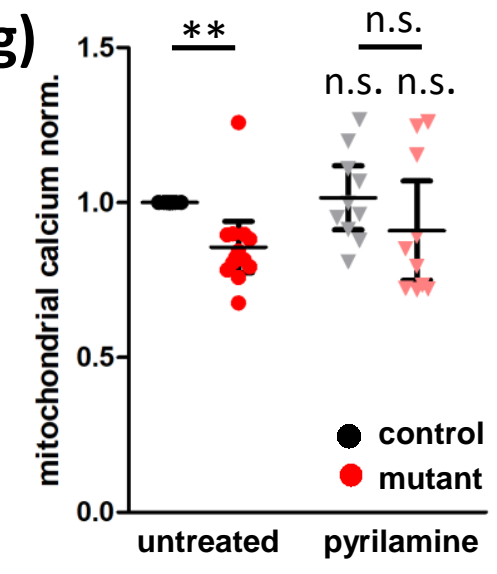

b)

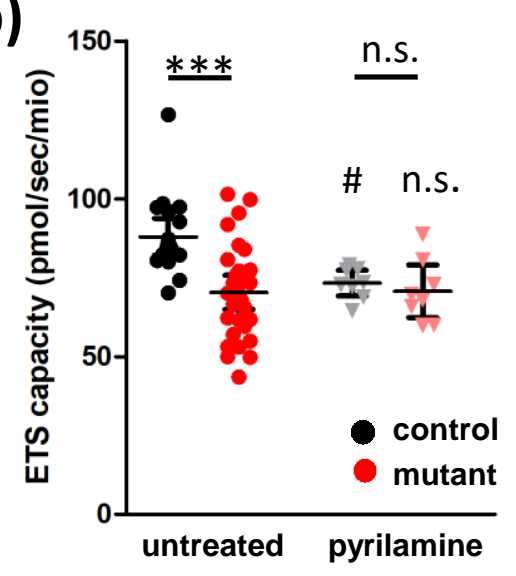

e)

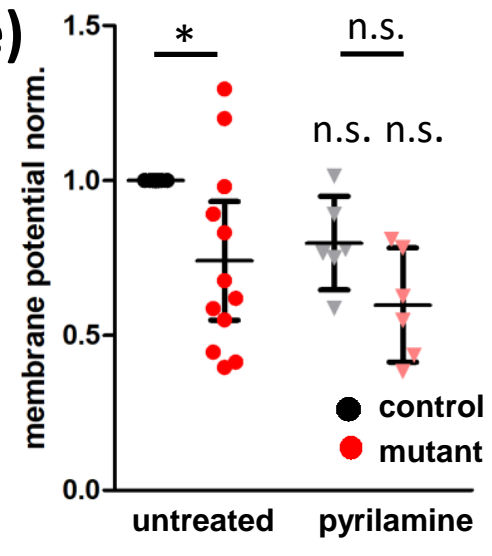

h)

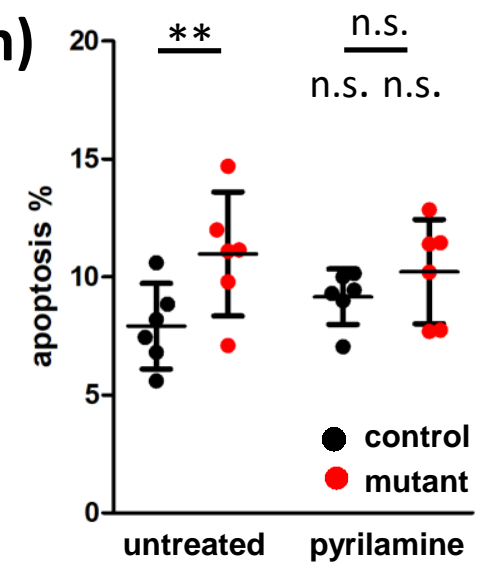

c)
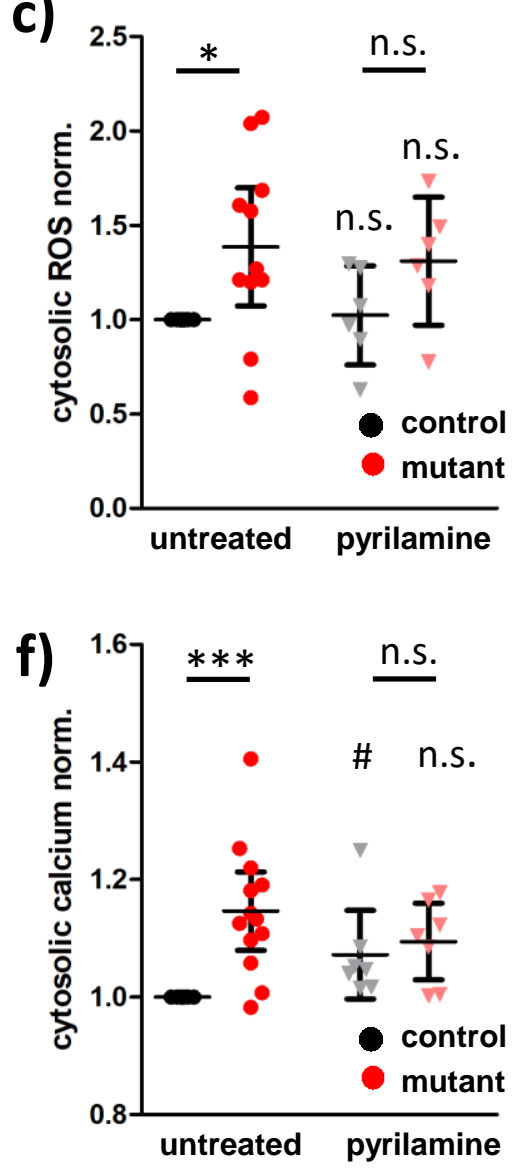


\section{Fig.S10 NR and NAC can partially rescue the mitochondrial defect in the ND5 m.13708G>A-H7 mtDNA cybrids}

a)

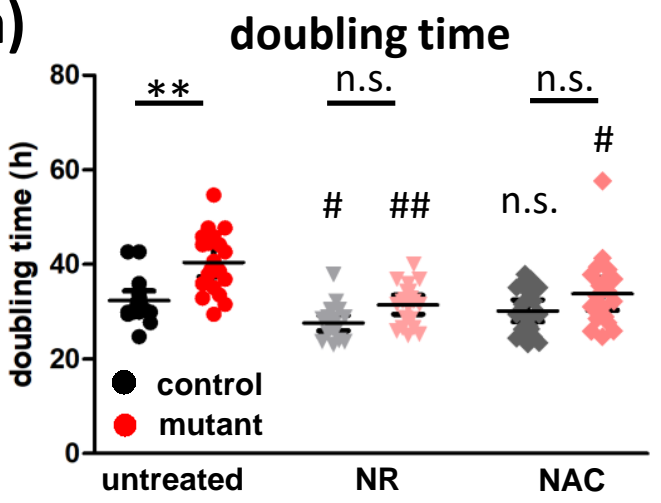

c)

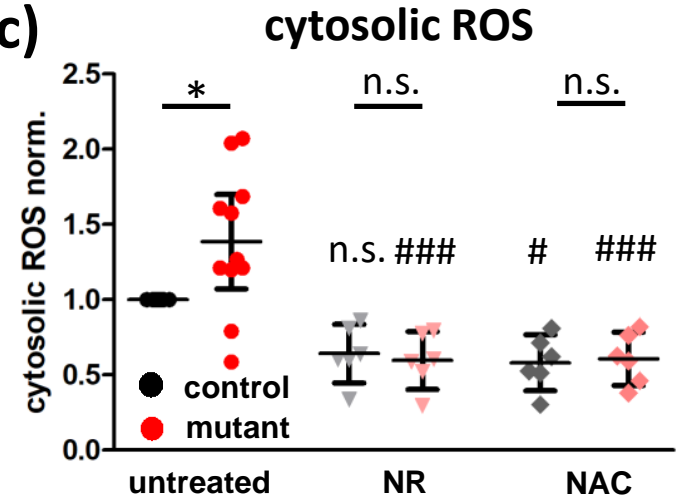

e)

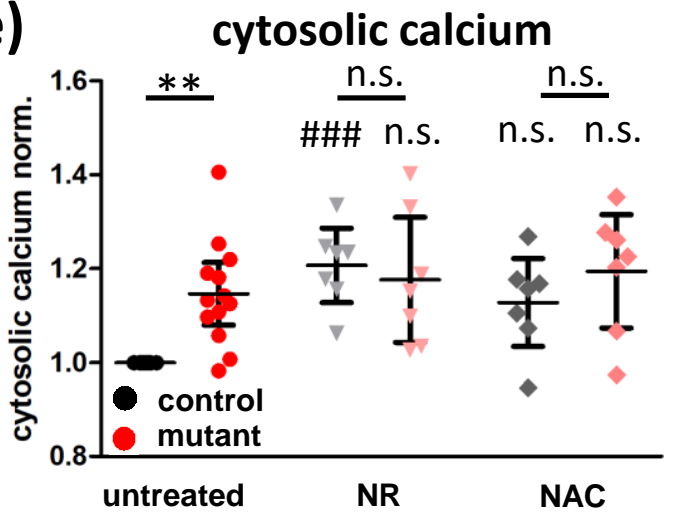

b)

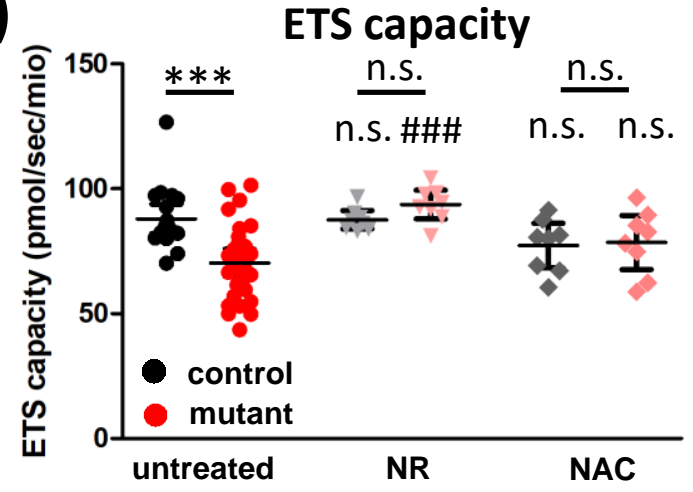

d)

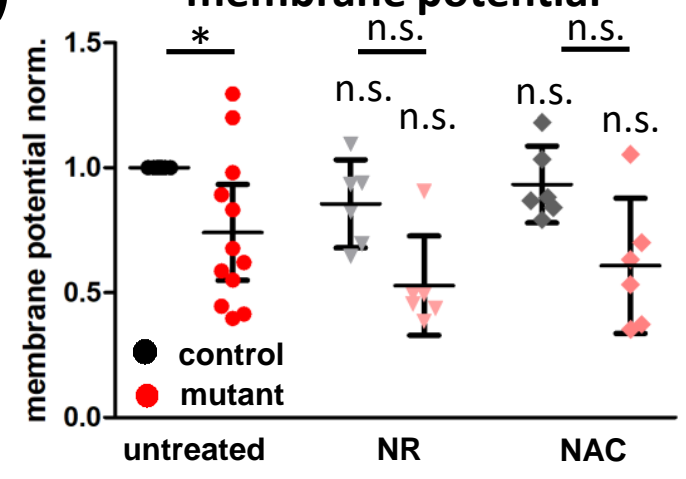

f)

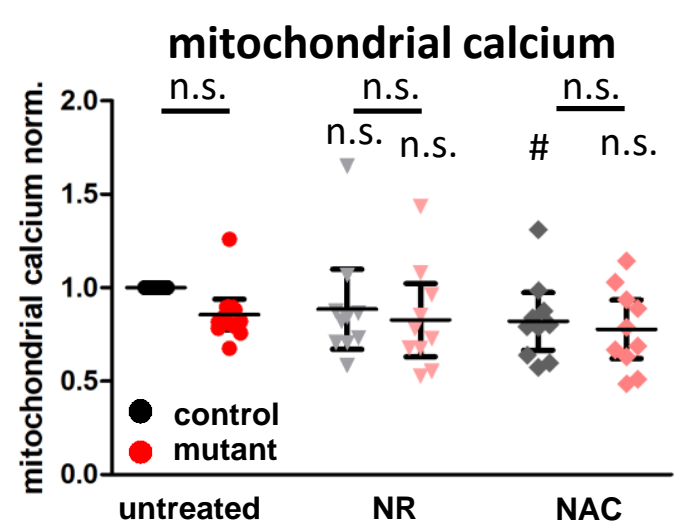




\section{Supplementary Appendix}

\section{Novel mtDNA Imparts the Connective Tissue Disorder of a Tourette Pedigree}

Patrick M. Schaefer, Dr. rer. nat. ${ }^{1}$, Leonardo Scherer Alves, Ph.D. ${ }^{1}$, Maria Lvova, M.D. ${ }^{1}$, Jessica Huang ${ }^{1}$, Komal Rathi, MSc ${ }^{2}$, Kevin Janssen ${ }^{1}$, Ph.D., Arrienne Butic ${ }^{1}$, Tal Yardeni, Ph.D. ${ }^{1}$, Ryan Morrow, Ph.D. ${ }^{1}$, Marie Lott, M.A. ${ }^{1}$, Kierstin Keller, C.G.C. ${ }^{1}$, Benjamin A. Garcia, Ph.D. ${ }^{3}$, Clair A. Francomano, M.D. ${ }^{4}$, Douglas C. Wallace, Ph.D ${ }^{1,5^{*}}$.

${ }^{1}$ Center for Mitochondrial and Epigenomic Medicine, Children's Hospital of Philadelphia, PA, 19104.

${ }^{2}$ Department of Biomedical Informatics, Children's Hospital of Philadelphia, PA, 19104

${ }^{3}$ Department of Biochemistry and Molecular Biophysics, Washington University School of Medicine, St. Louis, MO, USA

${ }^{4}$ Department of Medical and Molecular Genetics, Indiana University School of Medicine, Indianapolis, IN, USA

${ }^{5}$ Department of Pediatrics, Division of Human Genetics, Perelman School of Medicine, University of Pennsylvania, Philadelphia, PA, 19104.

* Corresponding author: wallaced1@chop.edu 


\section{Detailed clinical findings}

Summation of previous report (1) with augmented familial observations.

11: Male, age 48: (Tourette syndrome, Obsessive-Compulsive Disorder [OCD]). "history of motor tics including shoulder shrugs and neck stretching. Vocal tics included persistent clearing of the throat" (1).

12: Female, age 46 (connective tissue, metabolic alterations). "She has no history of tics, OCD, trichotillomania, or Attention Deficit Disorder (ADHD)" (1). Additional familial reported findings: pseudotumor cerebri (increased pressure around brain), joint hypermobility with post-compression detethering and decompression (tethered cord syndrome: tissue attachments that limit the movement of the spinal cord within the spinal column), chiari malformation (extension of brain into spinal cord, craniocervical mobility requiring fusion), hypermobility below cranio-cervical fusion, chronic diarrhea, ketosis, difficulties with temperature regulation, weight loss and nausea on Diamox (carbonic anhydrase inhibitor).

II1: Female, age 25 (Tourette syndrome, OCD, metabolic alterations). Aspergers (autism spectrum disorder), ADHD, OCD, hair pulling (trichotillomania), skin-picking (dermatillomania) (1). Additional familial reported findings: Low energy and frequent mild fevers.

II2: Male, age 23 (Tourette syndrome, connective tissue, and metabolic alterations). "Motor tics..." "Vocal tics consisted of throat clearing". "Type I diabetes, severe myopia, symptomatic chiari malformation, and tethered spinal cord", "joint hypermobility,..high, narrow palate.." "tricuspid and mitral valve" dysfunction, "diagnosed with a variant of EDS [Ehler-Danlos syndrome]" (1). Additional familial reported 
findings: skin herniation which spontaneously improved, possible ankylosing spondylitis (altered posture), kidney issues, DMI (diabetes mellitus insipidus).

II3: Male, age 21 (Tourette syndrome, connective tissue, and cardiovascular disorders). "mild clubbed feet", mild joint hypermobility", 'chiari malformation", "myopia, bicuspid aortic valve and root dilation", "sleep apnea" (1). Additional familial reported findings: Severe cranio-cervical instability, brainstem surgery, aortic aneurism (44mm) with oval aorta on MRI, syncope (loss consciousness) with exercise, supraventricular tachycardia and bradycardia, neurogenic bladder, and patches on retina.

I14: Male, age 18 (Tourette syndrome, connective tissue, and metabolic alterations). "cardiac involvement, pectus excavatum and high arced palate" (1). Additional familial reported findings: chiari malformation, dysautonomia, and tall (6'3").

II5: Female, age 16 (Tourette syndrome, connective tissue, metabolic and immunological alterations). "Type I diabetes, autonomic dysfunction (tachycardia and narrowed vision), severe joint pain, and symptomatic chiari malformation", "diagnosed with early-onset osteo-arthritis and a variant of EDS" (1). Additional familial reported findings: post-compression detethering and decompression, muscle weakness, kidney reflux, cranio-cervical instability, ankylosing spondylitis requiring Lidocaine infusions into spinal cord (assessment "life stinks"), dysautonomia, dehiscence (wound reopening post-surgery), unusual blood vessels brain to pelvis with stroke at age five, altered prothrombin time, multiple drug allergies, multiple infections including methicillin-resistant Staphylococcus aureus, Herpes, and Candida.

I16: Male, age 13 (Tourette syndrome, connective tissue, and metabolic alterations). "evaluated for probable connective tissue disorder with pectus excavatum and hypermobile joints.": Brain MRI revealed 
chiari malformation and hydromyelia syrinx", "surgical ... spinal cord detethering", "ketone utilization disorder"(1). Additional familial reported findings: episodic bradycardia, 3- $\beta$-keto-thiolase deficiency, high prolactin levels, carnitine bicitrate therapy.

II7: Male, age 10, monozygotic twin (Tourette syndrome and OCD, connective tissue, and metabolic alterations). "symptomatic chiari malformation", "high/arched palate, bilateral dislocation of hips, and moderate joint laxity", "mild convex cervical scoliosis centered at C6/C7", "sleep apnea and breathing pauses while awake", "possible connective tissue disorder at 6 years of age", "an EDS variant" (1). Additional familial reported findings: pseudotumor cerebri (increased pressure around brain) treated with ventriculoperitoneal shunt, left eye ptosis, joint hypermobility, post-compression detethering, and decompression, frequently falls asleep, chronically elevated CO2 while sleeping, apnea-hypoapea greater than 10 second, altered prothrombin time.

II8: Male, age 10, monozygotic twin (Tourette syndrome, connective tissue, cardiovascular and metabolic alterations)." described as having thin skin, easy bruisability, and joint laxity and dislocation. He has been diagnoses with a tethered spinal cord but has not required surgery", "bilateral strabismus with surgical correction" (1). Additional familial reported findings: prediabetic (blood sugar 110-112 mg/dL); hypermobile joints with subluxation (partial bone dislocation on CT) of knees, hips, and ankles with severe foot and leg pain; superficial veins on left arm; altered prothrombin time; left ventricular hypertrophy and hypertension starting at 8 years.

\section{References}


bioRxiv preprint doi: https://doi.org/10.1101/2022.02.25.481696; this version posted February 25, 2022. The copyright holder for this preprint

(which was not certified by peer review) is the author/funder, who has granted bioRxiv a license to display the preprint in perpetuity. It is made available under aCC-BY-NC-ND 4.0 International license.

1. Ercan-Sencicek AG, Stillman AA, Ghosh AK, Bilguvar K, O'Roak BJ, Mason CE, et al. L-histidine decarboxylase and Tourette's syndrome. N Engl J Med. 2010;362(20):1901-8. 